\title{
Video Microscopy Applied to Optical Fiber Geometry Measurements
}

Nathan Andrew Brilliant Bradley K. Alpert Matt Young

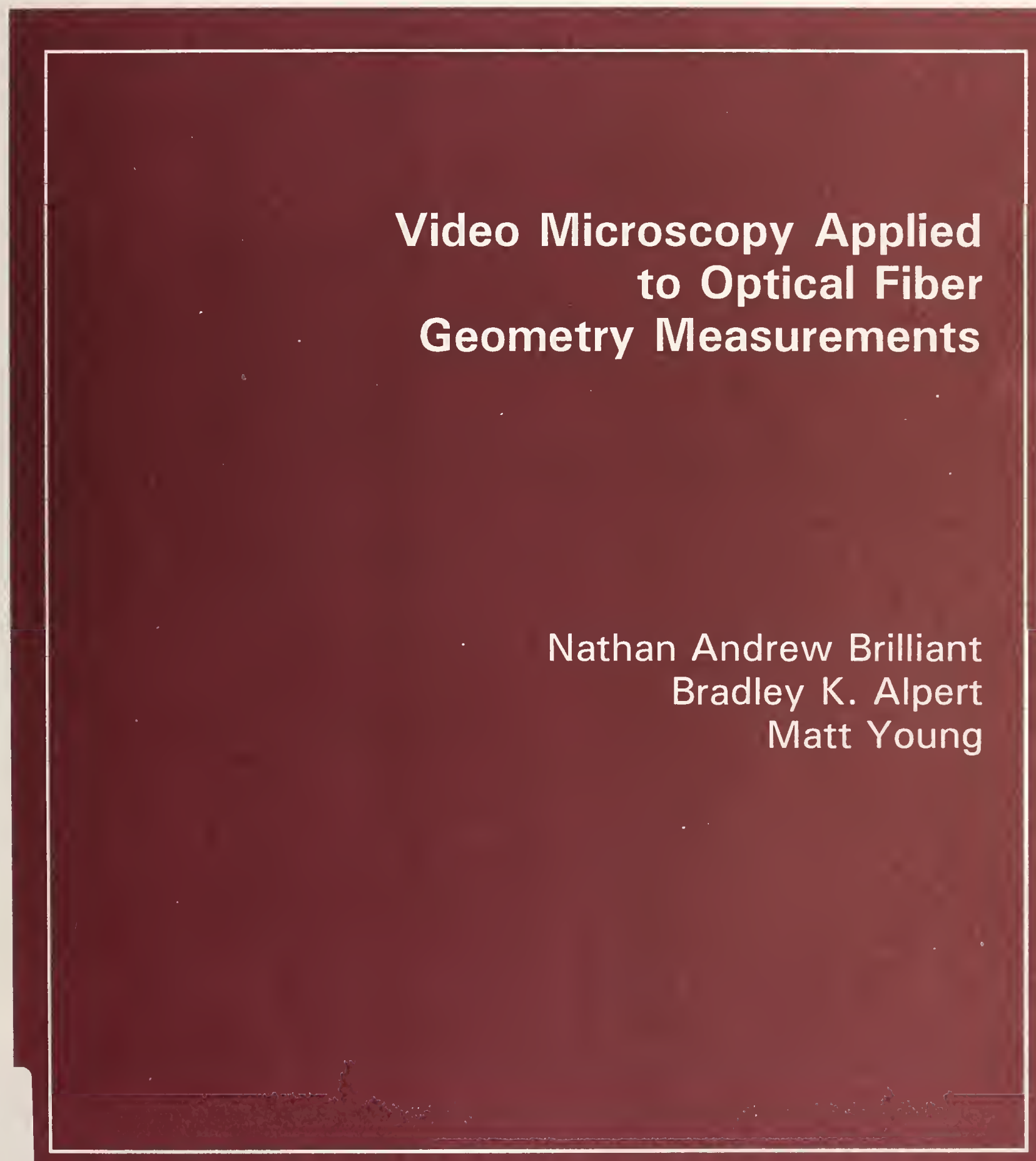





\title{
Video Microscopy Applied to Optical Fiber Geometry Measurements
}

\author{
Nathan Andrew Brilliant \\ Bradley K. Alpert \\ Matt Young
}

Optoelectronics Division

Electronics and Electrical Engineering Laboratory

325 Broadway

National Institute of Standards and Technology

Boulder, Colorado 80303-3328

November 1994

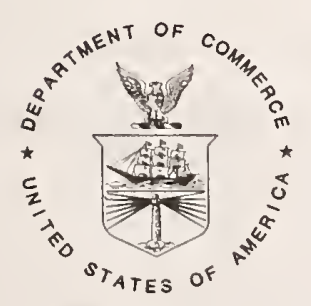

U.S. DEPARTMENT OF COMMERCE, Ronald H. Brown, Secretary TECHNOLOGY ADMINISTRATION, Mary L. Good, Under Secretary for Technology NATIONAL INSTITUTE OF STANDARDS AND TECHNOLOGY, Arati Prabhakar, Director 
National Institute of Standards and Technology Technical Note

Natl. Inst. Stand. Technol., Tech. Note 1369, 48 pages (November 1994) CODEN:NTNOEF

\section{U.S. GOVERNMENT PRINTING OFFICE \\ WASHINGTON: 1994}




\section{Contents}

1. Introduction $\ldots \ldots \ldots \ldots \ldots \ldots \ldots \ldots \ldots \ldots \ldots \ldots \ldots \ldots \ldots \ldots$

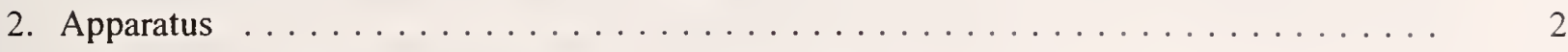

3. Calibration and Data Reduction ...................... 5

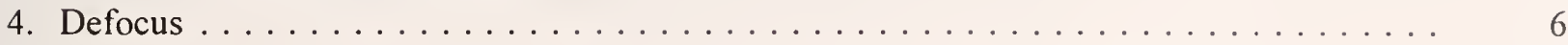

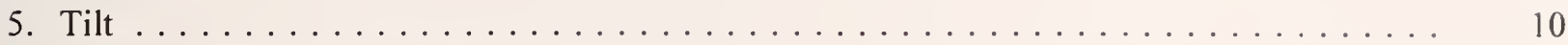

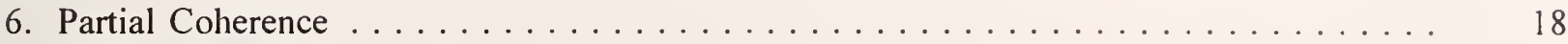

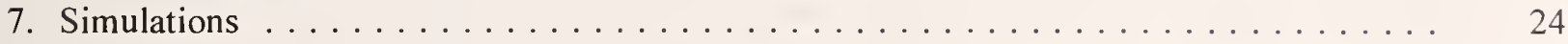

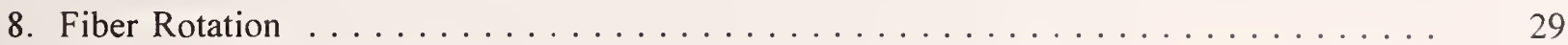

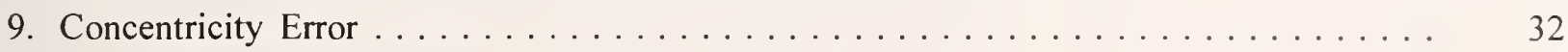

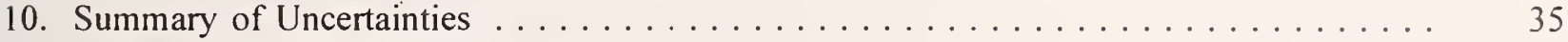

11. Other Fibers .............................. 36

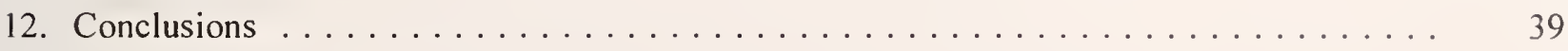

13. References ............................. 40

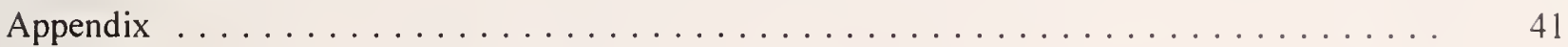




\section{Note}

Based in part on the Master's Thesis submitted by Nathan Brilliant to the Department of Physics of the University of Colorado at Boulder. Thanks to Dana Z. Anderson of the University of Colorado and Judah Levine of the Joint Institute for Laboratory Astrophysics for their careful reading of the thesis and their constructive criticism. 


\title{
Video Microscopy Applied to Optical Fiber Geometry Measurements
}

\author{
Nathan Andrew Brilliant, Bradley K. Alpert,* and Matt Young \\ Optoelectronics Division \\ National Institute of Standards and Technology \\ Boulder, Colorado 80303-3328
}

\begin{abstract}
We describe a video microscope used to measure the mean diameter and noncircularity of the cladding of optical fibers. We attempted, without success, to perform absolute measurements. When we calibrated the microscope with a fiber of known diameter, however, we could measure the cladding diameter within $0.1 \mu \mathrm{m}$.
\end{abstract}

Key words: coherence; gray scale; imaging; micrometer; microscope; optical fiber geometry; optical fibers; video microscopy

\section{Introduction}

This Technical Note describes a measurement of the mean diameter and noncircularity of the cladding of optical fibers, more specifically, an attempt to perform the measurement absolutely with a video microscope. Engineers must have accurate measurement of both mean diameter and noncircularity to make precise, low-loss connectors. The industry requires a device which can measure the mean cladding diameter with an accuracy of $0.1 \mu \mathrm{m}$. The video microscope is the most common instrument for making measurements of fiber geometry.

The most accurate measurements are made with interferometric microscopes and contact micrometers. In our division, we use a contact micrometer to make diameter measurements accurate within $40 \mathrm{~nm}$ [1]. We manufacture Standard Reference Material (SRM) fibers for the telecommunications industry so that they have calibration artifacts for their measuring instruments. The SRMs are approximately $125 \mu \mathrm{m}$ in diameter and are very nearly circular.

We measured a number of fibers with the micrometer and used them to check our measurements. we did not succeed in making the video microscope an absolute device: Our mean diameter measurements were consistently high by $0.19 \mu \mathrm{m}$. Nevertheless, the $3 \sigma$ uncertainty in our measurements is $0.07 \mu \mathrm{m}$, which is better than the required accuracy. Thus, the microscope provides the necessary accuracy, but only if calibrated with an artifact such as the SRM. We also measured the noncircularity and the position of the major axis of an ellipse fitted to the edge of the fiber.

The video microscope has a number of inherent problems. As with all optical equipment, the focus of the instrument is critical. Slight defocusing can greatly change the apparent diameter of the optical fiber being viewed. Because of the depth of focus of the instrument, it is difficult to find the precise focus. Sensitivity to focus also creates a problem when the fiber end face is not perpendicular

*Applied and Computational Mathematics Division, Computing and Applied Mathematics Laboratory 
to the optical axis of the microscope. When the end is tilted, parts of the fiber are in focus while other parts are not in focus. This creates the illusion of a noncircular fiber end. Even when the image is correctly focussed, the image is not sharp because of diffraction or aberrations.

The width of the image of the edge is on the order of $1 \mu \mathrm{m}$ as a result of diffraction by the aperture stop of the objective. To find the intensity which corresponds to the edge of the fiber, we must use the theory of partially coherent light. The width of the edge profile and the circular nature of the fiber end combine with the rectangular geometry of the pixels in the ccd camera to create a systematic deviation of the apparent edge. Precise characterization of the deviation could result in a more accurate location of the edge of the fiber.

\section{Apparatus}

We inherited the microscope used by Steven Mechels [2] and Lakshmikant Mamileti [3].

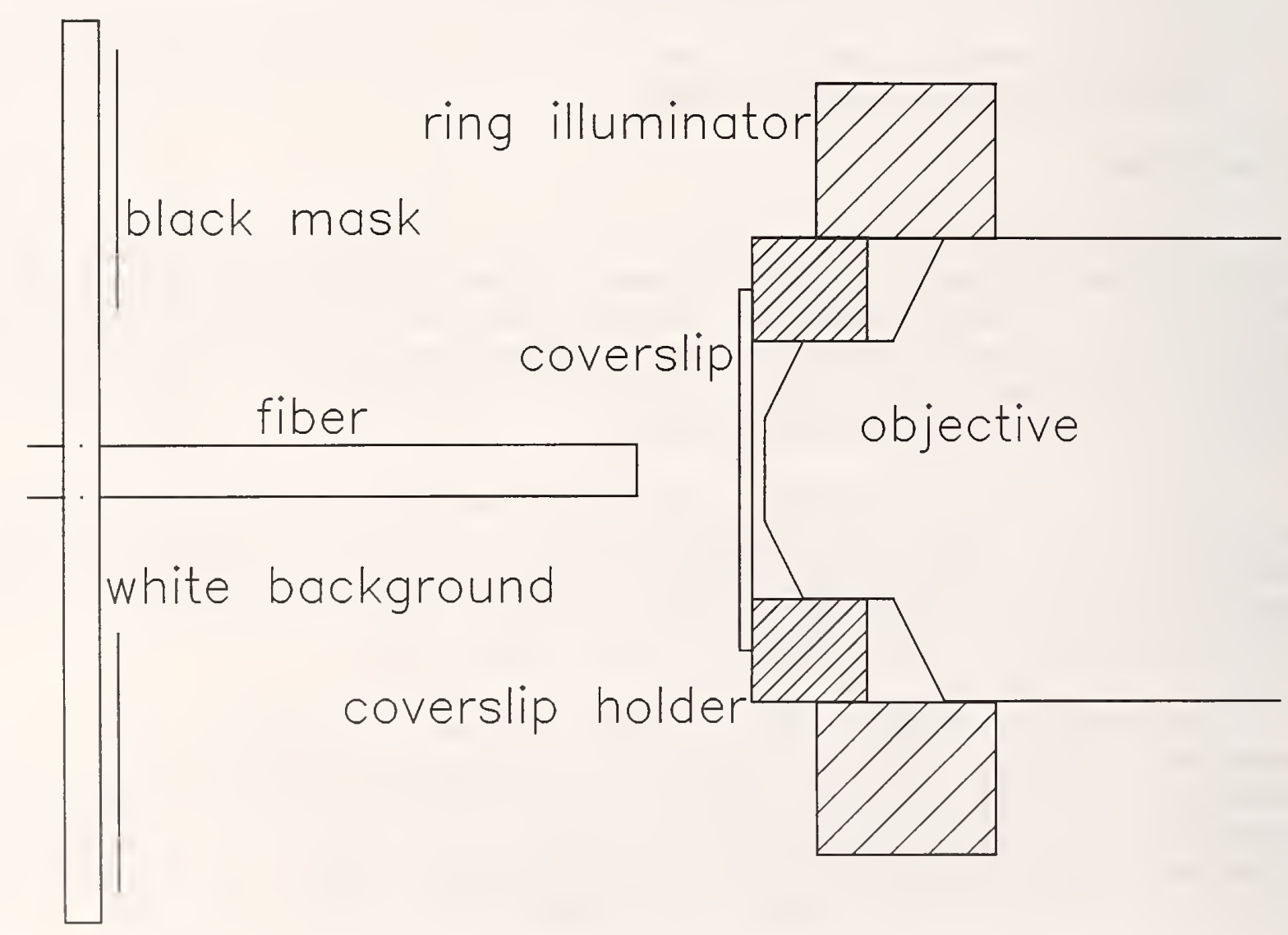

Figure 1. Detail of illuminator and microscope objective.

The microscope itself consists of an objective lens and a ccd camera connected by a metal tube. Inside the metal tube are two glare stops. Four hardened steel rods rigidly hold the objective, tube, and camera. The objective has a numerical aperture of 0.65 and a magnification of $40 \times$. We chose the $40 \times$ 
magnification to ensure that the image of the fiber would fill about $80 \%$ of the available pixel array in the camera.

The objective is designed to function with a coverslip. Use of the objective without a coverslip results in spherical aberration [4], so we attached a coverslip to a cylinder of phenolic. The cylinder fits over the object and inside the ring illuminator as shown in Figure 1.

The camera is a frame transfer array as discussed in [5]. It has 512 pixels horizontally and 220 vertically. The pixels are rectangular and measure $11 \mu \mathrm{m}$ horizontally and $27 \mu \mathrm{m}$ vertically. Because of the way the microscope is calibrated, the absolute dimensions of the pixels are not important.

The camera reads the information in the pixels one line at a time. A set of 220 lines is called a field. Our camera operates in the interlaced mode: it reads two separate fields. One field consists of lines corresponding to the pixels themselves. The second field consists of lines of pseudopixels. Each pseudopixel is centered vertically between the real pixel above and the real pixel below. The light-gathering area of the pseudopixel is half of the pixel above and half of the pixel below. The camera achieves this second field by biasing the pixels. The net image is a $512 \times 440$ array called a frame.

The output of the camera goes to a commercially available frame digitizer running on a personal computer and to a video monitor. The resulting array assigns values between 0 and 255 for each pixel. We use the monitor to visually position and focus the image of the fiber end. Light from a ring illuminator reflects from a plate behind the fiber to back-illuminate it. The ring illuminator has a hole drilled through its center and fits snugly around the objective. Instead of locating a green filter between the objective and the camera (that is, inside the microscope) [2], we drilled a hole in a green filter which passes a band of light centered at $525 \mathrm{~nm}$ and attached it to the ring illuminator. We use a narrow band to reduce chromatic aberration. Attaching the filter to the ring illuminator allows us to illuminate the core with light of any wavelength.

The fiber is held in place by a three-axis optical stage. The stage has micrometers to control position along the optical axis of the microscope as well as two orthogonal directions perpendicular to the optical axis. We will call the axis parallel to the optical axis the $\mathrm{z}$ axis and the perpendicular directions the $\mathrm{x}$ and $\mathrm{y}$ axes. The $\mathrm{x}$ axis is parallel to the floor. The perpendicular controls position the fiber in the center of the field of view of the microscope. The parallel controls focus the image of the fiber. There are two micrometers for focus control. One micrometer is marked in $25 \mu \mathrm{m}(0.001$ in) increments, the second in $1 \mu \mathrm{m}(40 \mu \mathrm{in})$ increments.

To accommodate an SRM fiber, we attached a machined aluminum box to the back of the white aluminum plate. The SRM is secured in the box by two screws. The fiber can be extended through a hole in the aluminum plate. The plate reflects light as a source of back-illumination. The plate is fixed to a beam splitter mount which is attached to the optical stage, as shown in Figure 2. 


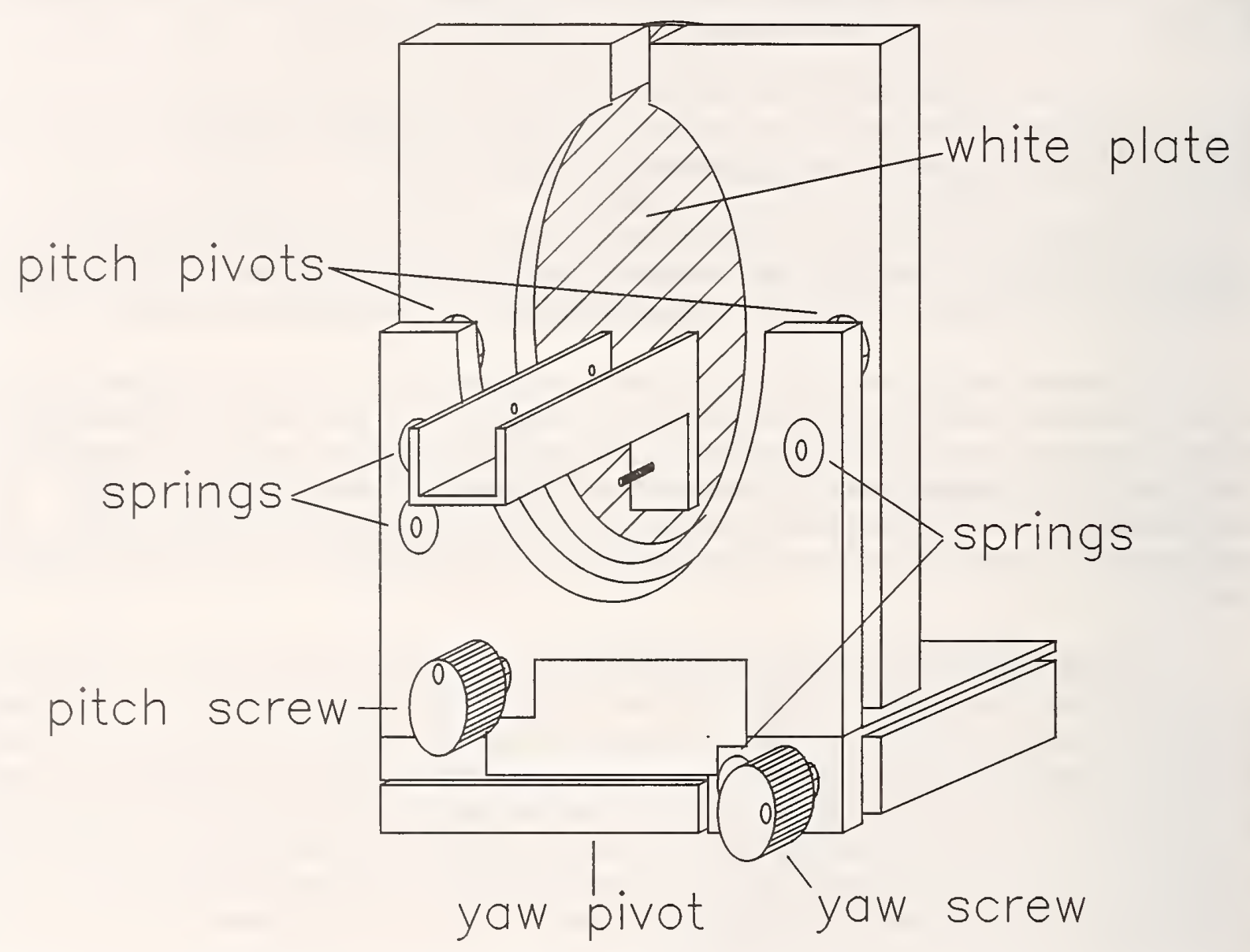

Figure 2. Beam splitter mount and white plate.

The beam splitter mount provides control over the relative angle between the plane of the fiber end and the optical axis. The two possible rotations are about the $\mathrm{x}$ axis (pitch) and the $\mathrm{y}$ axis (yaw). We modified the system by attaching a black mask to the aluminum plate. To fix the size of the illumination spot, we punched a $0.95 \mathrm{~cm}$ diameter hole in the center of the mask. The hole in the mask is concentric with the hole in the metal plate. We punched the hole in the mask both to fix the size of the illumination spot and to make it independent of the position of the ring illuminator.

An SRM fiber is enclosed in an aluminum box, or SRM holder, to protect it. The fiber is glued into a ferrule in a brass barrel; the barrel itself rests in the aluminum box. The front of the box has a hole though which the fiber projects. The barrel slides forward and backward to project and retract the fiber end. The barrel is marked in $45^{\circ}$ increments and rotates inside the box. All of the fibers were mounted in SRM holders.

We also illuminate the core of the fiber to investigate its geometry. We use a tungsten lamp, a lens, and a second microscope objective to focus light into the core. We wrap the fiber around a mandrel to scramble the modes. The fibers used are single-mode fibers for $1.3 \mu \mathrm{m}$ light; they are multimode at visible wavelengths. Multimode operation gives rise to a nonuniform intensity profile in the core; this can create an error in measuring the positions of the edge and the center of the core. Wrapping the fiber about a mandrel scrambles the modes and causes a more uniform intensity profile. 
Figure 9 is the angle of the major axis as a function of tilt. Yaw draws the major axis toward $-90^{\circ}$ (vertical) whereas pitch draws it toward $0^{\circ}$ and $-180^{\circ}$ (horizontal).

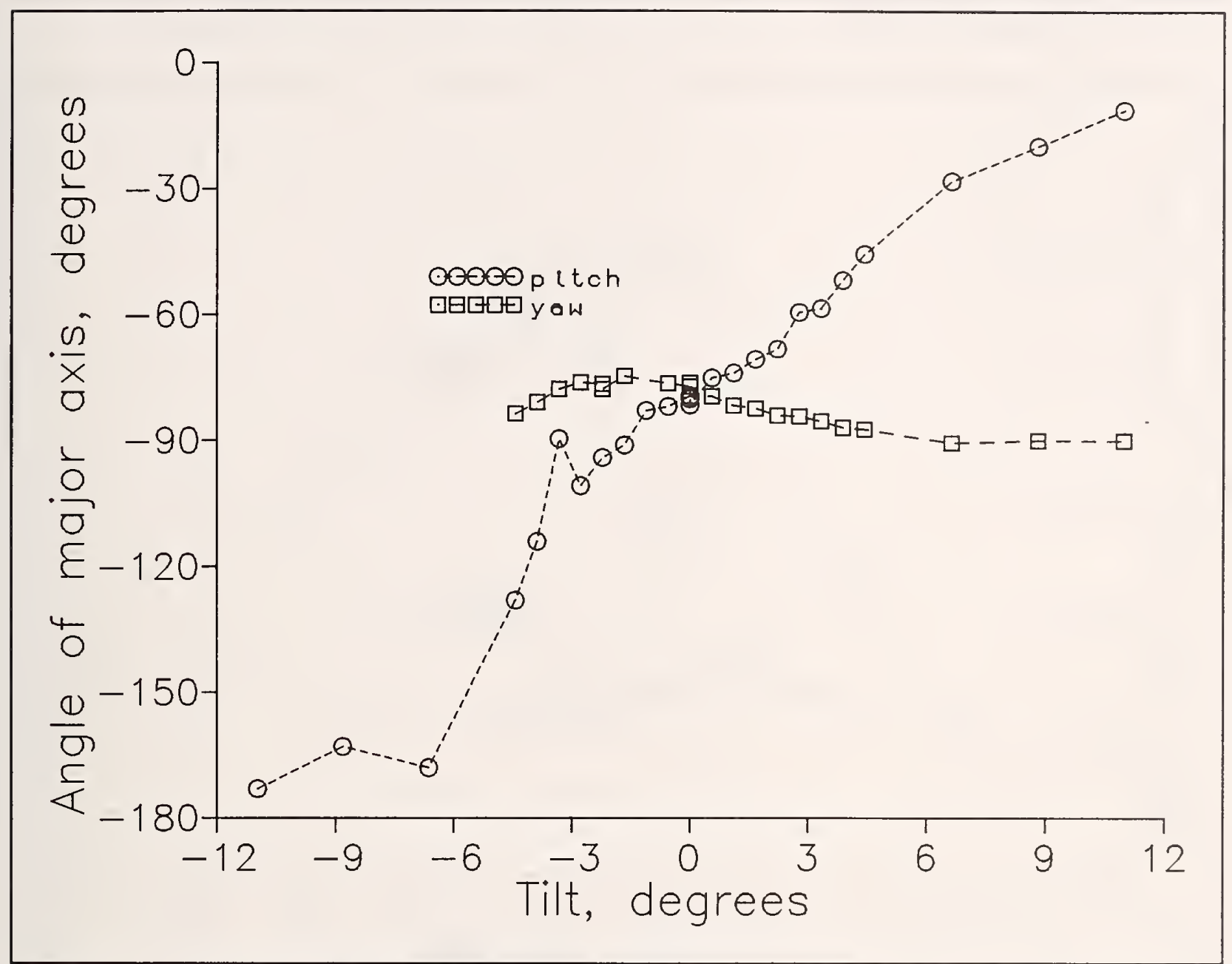

Figure 9. Major axis angle as a function of tilt angle.

For the second set of data, we used a more severely noncircular fiber (Figures 10,11, and 12). The contact micrometer measured the mean diameter as $125.522 \mu \mathrm{m} \pm 0.04 \mu \mathrm{m}$, the noncircularity as $0.25 \%$, and the angle as $-89^{\circ}$. These data also support the suppositions. 
The graph of mean diameter as a function of tilt angle in Figure 10 is less smooth than that in Figure 7. The point labeled $0^{\circ}$ yaw on the graph is no longer $0^{\circ}$ yaw, possibly because the yaw screw was very loose and near the end of its travel. As in Figure 7, an increment of yaw has greater effect than an equal increment of pitch.

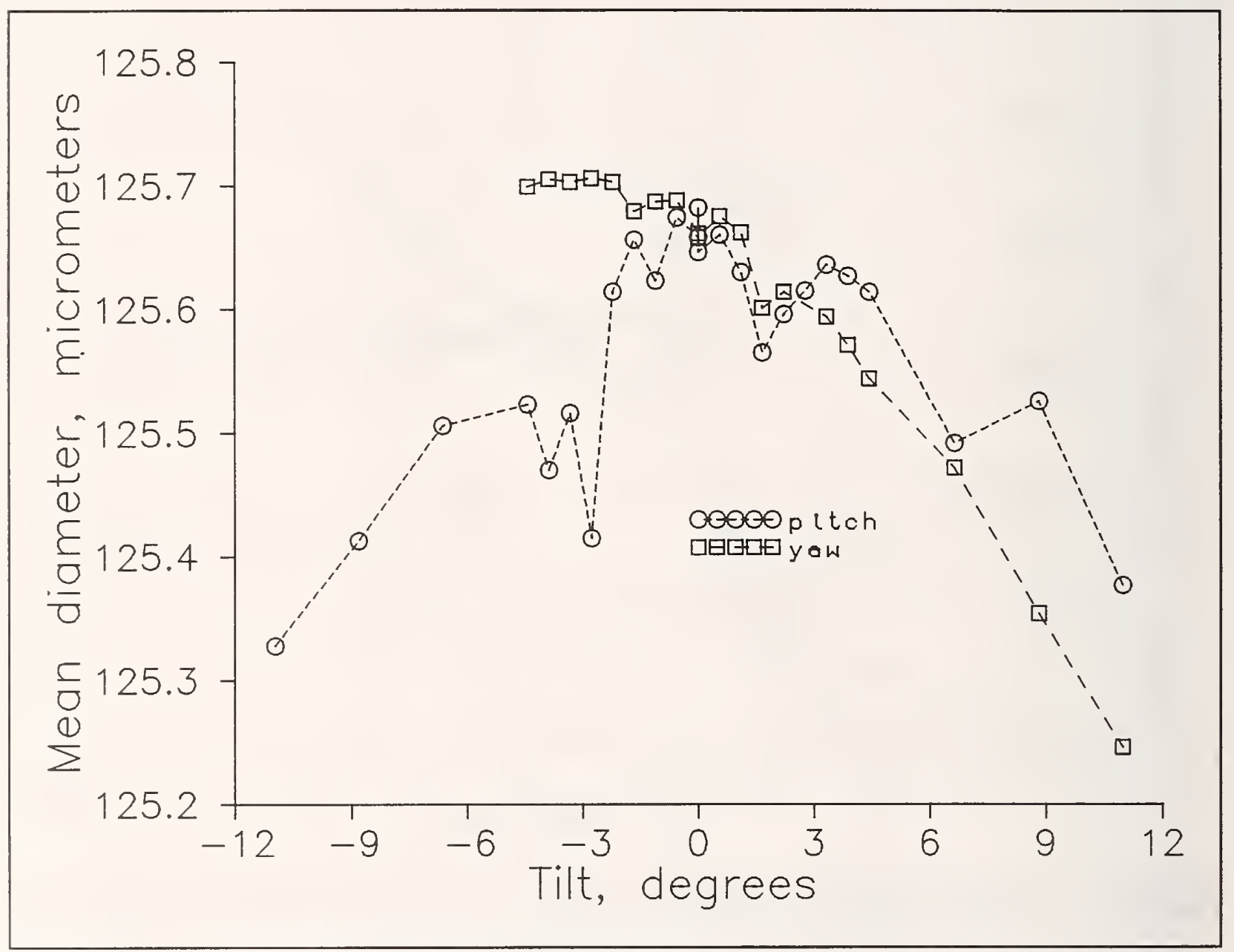

Figure 10. Mean diameter as a function of tilt angle. 
The noncircularity in Figure 11 has a minimum at $0^{\circ}$ yaw. It has a local maximum at $0^{\circ}$ pitch and local minima at $9^{\circ}$ and $-6^{\circ}$. Just as for Figure 8, these are the results we expect, since the major axis of the fiber is near $-90^{\circ}$. At the local minima, the vertical diameter and the horizontal diameter are the same; this places the major axis near $-45^{\circ}$ or $-135^{\circ}$.

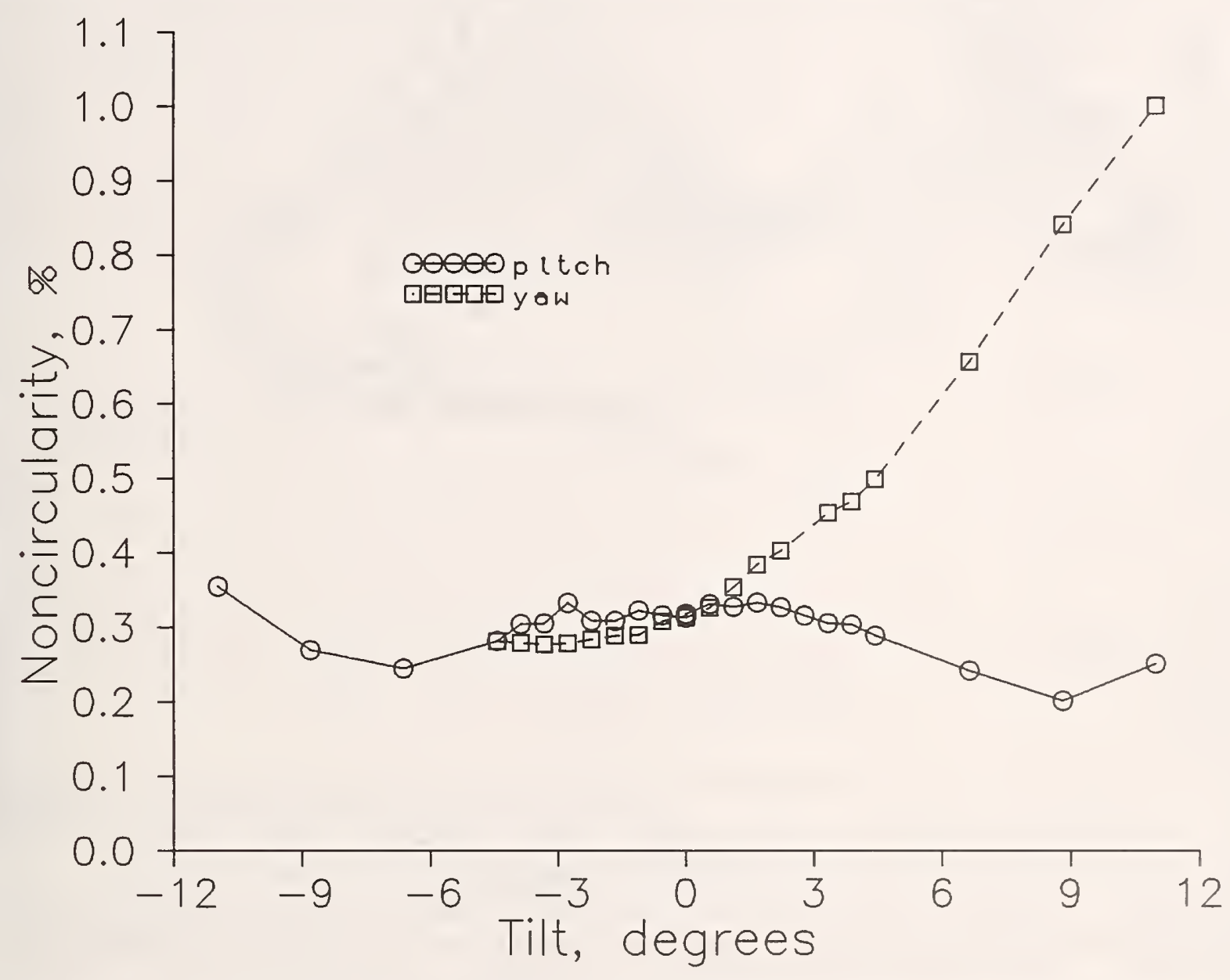

Figure 11. Noncircularity as a function of tilt angle. 
Figure 12 shows that the angle of the major axis is $-60^{\circ}$ at $9^{\circ}$ pitch and $-120^{\circ}$ at $-6^{\circ}$ pitch. These are close to the predictions of $-45^{\circ}$ and $-135^{\circ}$. For larger pitch angle, the major axis is drawn to the horizontal. Yaw does not affect the direction of the major axis since it was already nearly $-90^{\circ}$.

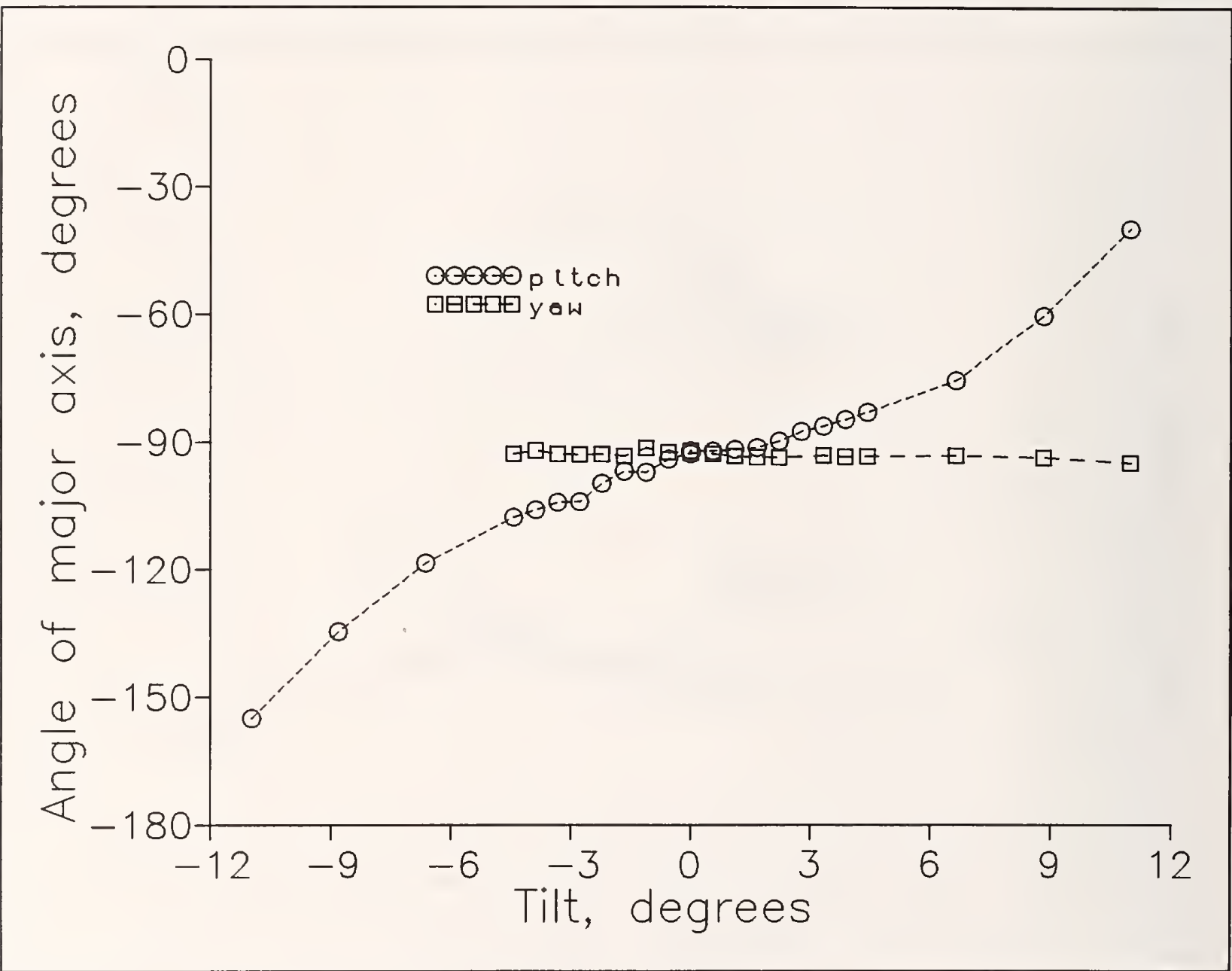

Figure 12. Angle of major axis as a function of tilt angle.

The effects of tilting the fiber do not change as the fiber rotates about its axis. This allows us minimize the tilt. By looking at an edge table in polar coordinates, we can determine whether the angle of the major axis rotates as the fiber rotates.

In a plot of radius as a function of angle, a circular fiber appears as a horizontal line because the radius is independent of angle. An elliptical fiber appears as a sinusoid. The two maxima correspond to the major axis; the two minima correspond to the minor axis. Figure 13 is a plot of radius as a function of angle for a very nearly circular fiber. 


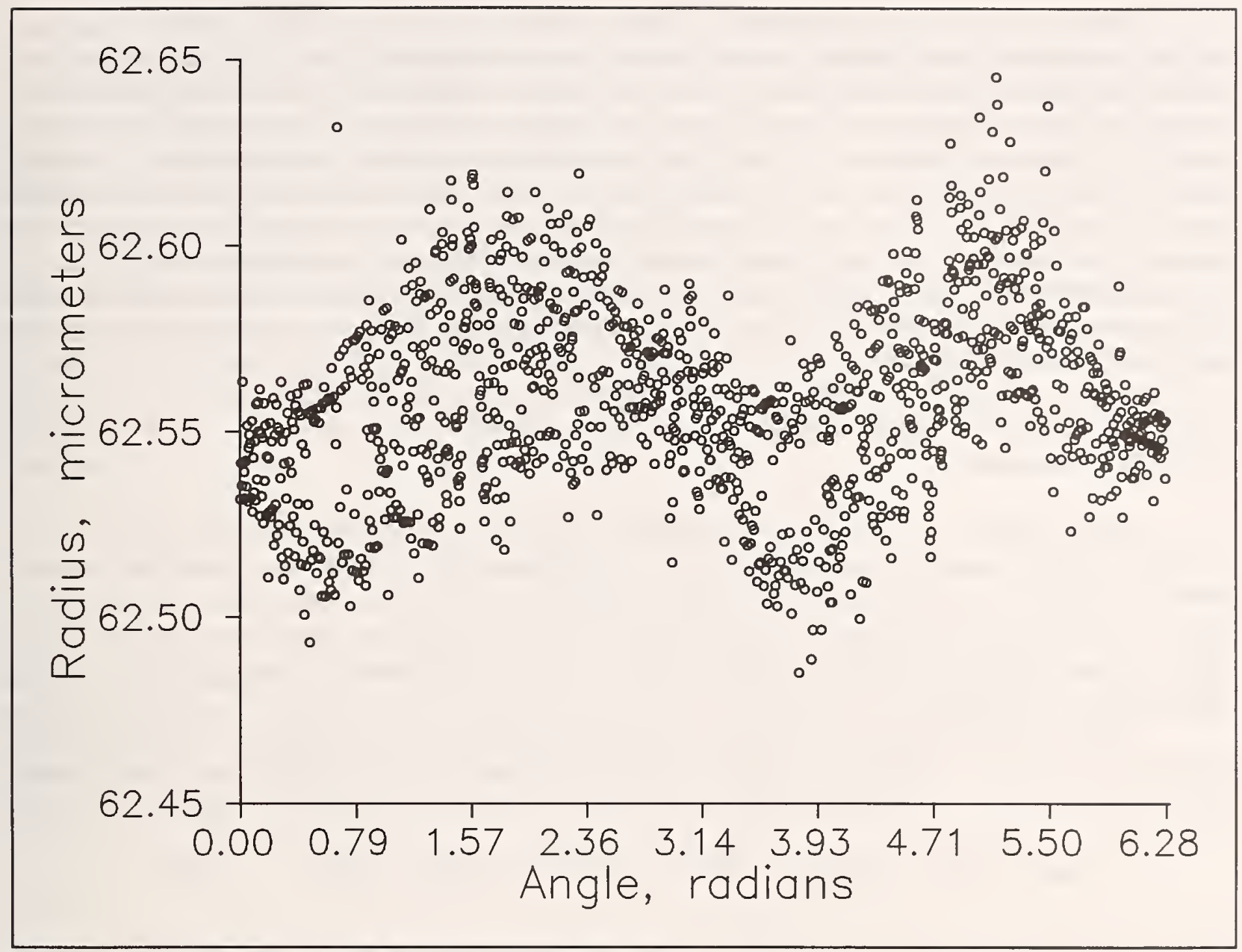

Figure 13. Radius of fiber as a function of angle.

Ideally, if the fiber rotates by $45^{\circ}$, then the maxima and minima should also shift by $45^{\circ}$. The magnitudes of the maxima and minima are determined by the noncircularity of the fiber. Tilting causes a second sinusoid to be added to the noncircularity sinusoid. The maxima and minima from the tilt sinusoid do not rotate with the fiber.

If there is pitch, the maxima for the second sinusoid appear at $0^{\circ}$ and $180^{\circ}$. If there is yaw, the maxima are at $90^{\circ}$ and $270^{\circ}$. If, as the fiber rotates, there are persistent maxima at $90^{\circ}$ and $270^{\circ}$, yaw must be adjusted. If the persistent maxima appear at $0^{\circ}$ and $180^{\circ}$, pitch must be adjusted. By checking the movement of the maxima, we can adjust pitch and yaw to $0^{\circ}$ within $1^{\circ}$ or so. Inside this range, we can no longer distinguish between two edge tables.

It is difficult to determine the precise effect of tilt on the mean diameter (Figures 7 and 10). The uncertainty due to defocus is so large that it overwhelms the effect of tilting. Therefore, we fitted a parabola to the mean diameter as a function of tilt angle. The value of the fitted curve drops by $0.004 \mu \mathrm{m}$ when the tilt angle is $1^{\circ}$. We will assume that the best value is at the middle of this range and that the distribution about the best value is Gaussian. If we assume the $0.004 \mu \mathrm{m}$ range to be $6 \sigma$ then the $1 \sigma$ uncertainty is $\pm 0.0007 \mu \mathrm{m}$. The range of the noncircularity is about $0.04 \%$ over a $2^{\circ}$ range; again we will assume that this range is $6 \sigma$. The $1 \sigma$ uncertainty of the noncircularity due to tilt is thus $\pm 0.007 \%$. The range in the angle of the major axis is $8^{\circ}$; the $1 \sigma$ uncertainty is $\pm 1.3^{\circ}$. 
However, the beam splitter mount is not the only possible source of tilt. Cleaving of the fiber end does not always leave a surface perpendicular to the axis of the fiber. When we cleave the fibers, we check the flatness and perpendicularity of the ends with a commercial Michelson interferometer that uses $730 \mathrm{~nm}$ light. The best ends display about three fringes; this implies an angle of about $0.5^{\circ}$, which is enough to alter the appearance of the fiber in the video microscope. It is impossible to tell the relative geometry between the tilt of the cleave and the actual major axis of the fiber. The tilt axis could be parallel to the major axis of the fiber, perpendicular to the major axis, or somewhere in between. Because this tilt is part of the fiber end, we cannot adjust for it. The best we can do is to try to align the fiber with the optical axis. The uncertainty due to the cleave angle is about the same as that due to the tilt of the beam splitter mount, since we cannot determine the geometry of the tilt angle and the true major axis.

\section{Partial Coherence}

Even a perfectly focused image is not perfectly sharp due to diffraction by the aperture stop of the objective; this causes the image of the edge to have a finite width. The camera records the intensity pattern of the edge in the image plane of the microscope which we call the edge profile. The width of the edge profile makes it unclear where the true edge lies. To calculate the relative intensity at the edge, we used the theory of partially coherent light discussed by Beran and Parrent [8], Born and Wolf [9], and Goodman [10].

The ccd detects the time-averaged intensity; intensity is the square of the complex electromagnetic field. Consider two point sources of radiation. The total field at any point in space is the sum of the fields due to the two points. The ccd sees the time average of the square of the sum,

$$
I_{\text {time avg }}=\left\langle\left|E_{1}+E_{2}\right|^{2}\right\rangle=\left\langle\left|E_{1}\right|^{2}\right\rangle+\left\langle\left|E_{2}\right|^{2}\right\rangle+\left\langle E_{1} \bar{E}_{2}+\bar{E}_{1} E_{2}\right\rangle \text {. }
$$

When the square is expanded, the first two terms are then intensities due to the individual sources. However, there is also a cross term. If there is a fixed phase relationship between the fields due to the two points, then the time-averaged cross term can have a value other than 0 . The cross term will be a maximum if the fields are in phase and 0 if they are out of phase. The light is called coherent, and we must work with the electromagnetic field. If, on the other hand, there is no correlation between the phases of the two fields, then the time average causes the cross term to vanish. This is called the incoherent case, and we add the individual intensities to get the final intensity.

Unfortunately, for extended sources imaged through finite apertures, the light is neither coherent nor incoherent but is partially coherent. The convenient quantity is neither the electromagnetic field nor the intensity but the mutual coherence function

$$
\Gamma(x, y ; w, z ; \tau)=\langle V(x, y, t+\tau) \bar{V}(w, z, t)\rangle .
$$

Partially coherent light is linear in the mutual coherence function. $\mathrm{V}$ is the complex scalar field at a point $(x, y)$. The point $(w, z)$ is in the same plane as the point $(x, y)$. The bar indicates the complex conjugate. $\tau$ is a time difference. The $\diamond$ indicate a time average in $t$. The intensity is the mutual coherence function at a single point with $\tau=0$; that is,

$$
I(x, y)=\Gamma(x, y ; x, y ; 0) .
$$


The mutual coherence function satisfies two wave equations, one for each set of spatial coordinates. Therefore, if we know the mutual coherence function in the object plane and the Green's function (transfer function) of the optical system, we can find the mutual coherence function in the image plane. The Green's function for the mutual coherence function in one set of spatial variables is the same as the Green's function for the electromagnetic field under coherent illumination. With the mutual coherence function in the image plane, we can find the intensity. Both coherent and incoherent illumination can be described as certain limits of partially coherent illumination.

The mutual coherence function of a circular, mutually incoherent source can be described by a characteristic distance. A mutually incoherent source is one in which there is no correlation between the phases of the fields arising from any two points. In other words, the mutual coherence function of the source is a delta function of position. The characteristic distance is the distance between the maximum and first zero of the mutual coherence function. This distance, the radius of coherence, is determined by the numerical aperture of the source. In the paraxial approximation, the radius of coherence is

$$
r_{c}=0.61 \frac{\lambda}{N A_{s}}
$$

where $\mathrm{NA}_{\mathrm{s}}$ is the numerical aperture of the source. $\mathrm{NA}_{\mathrm{s}}$ is the sine of the angle formed by the optical axis and a line from the fiber end to the edge of the source. In Figure 14, $N A_{s}=\sin (a)$. The phases of points inside this distance are highly correlated. 


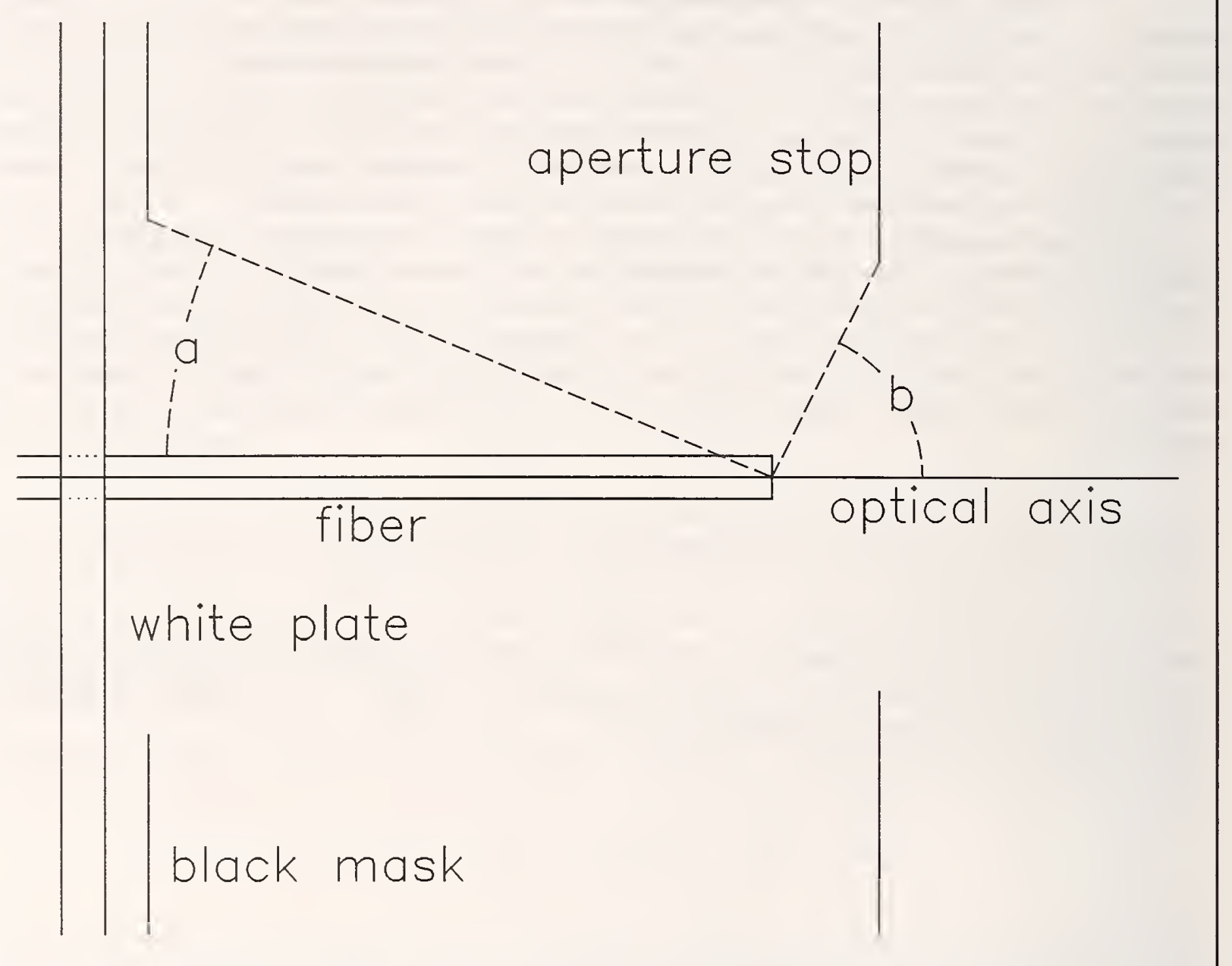

Figure 14. Numerical apertures.

The resolution limit of the microscope is a second characteristic distance which influences the degree of coherence. The resolution limit is the minimum distance between two equally intense point sources that appear as separate points in the image. The resolution limit in the object plane is defined as

$$
R L=0.61 \frac{\lambda}{N A_{o}}
$$

where $\mathrm{NA}_{\mathrm{o}}$ is the numerical aperture of the objective. In Figure $14, \mathrm{NA}_{\mathrm{o}}=\sin (\mathrm{b})$.

The degree of coherence of the system is determined by these two distances. If the coherence length is much larger than the resolution limit, then the system appears to be highly coherent. If the coherence length is much smaller than the resolution limit, then the system appears incoherent.

We define the coherence parameter $\alpha[11]$ as the ratio of the resolution limit to the coherence length or, equivalently, the ratio of the numerical aperture of the source to the numerical aperture of the microscope objective. A coherent source is a point source and has a coherence parameter of 0 . By contrast, a source can be considered incoherent only if the objective has a numerical aperture of 0 . An 
We use white light to illuminate the core to increase the number of modes; this also causes a more uniform intensity profile in the core. We cannot illuminate the core with a single symmetric mode because the ccd is not sensitive to $1.3 \mu \mathrm{m}$ light.

bench.

All of the apparatus except the computer and the video monitor is held in line by an optical

\section{Calibration and Data Reduction}

To measure diameter accurately, the optical system must be calibrated. That is, we need to determine the correspondence between numbers of pixels in the camera and distance in the object plane. We calibrated the microscope by using a chromium-on-glass reticle. The reticle has 21 clear lines on an opaque background. The lines are approximately $10 \mu \mathrm{m}$ apart. The reticle had been sent previously to NIST Gaithersburg for accurate measurement of the line separations.

To calibrate horizontally, we digitize an image with the lines running vertically on the screen. They are separated by spaces in the $\mathrm{x}$ direction. The image has 15 peaks in intensity [2]. We calculate the distance in pixels between the lines by calculating the distance between the peaks. Because each of the peaks has a finite width and is nearly symmetric about its center, we find the center of the line by finding the center of the segment between the half-maximum intensity points. The distance between two lines is the distance between their centers. By fitting a polynomial in pixel number to the data, we get distance as a function of pixels. Only the linear term is significant; it is a scale factor in micrometers per pixel. We repeat the same procedure with the lines rotated by $90^{\circ}$ for the vertical calibration. The calibration is described in more detail in [2]. The second term of the polynomial fit discussed in that reference turns out to be unreproduceable; its inclusion results in a distorted fiber image.

The final output of this system, a $512 \times 440$ array of hexadecimal numbers, represents the image of the fiber end in 256 gray levels. Jack Wang and Dominic Vecchia of the Statistical Engineering Division at NIST wrote the software which locates the edge and fits an ellipse to the edge [3].

The output of the program contains the Cartesian coordinates of the points on the edge, the coordinates of the center of the ellipse, the semi-major axis, the semi-minor axis, and the angle between the semi-major axis and the horizontal axis, as well as the same quantities for the core of the fiber.

The Cartesian coordinates of the points on the edge are called an edge table. There is an edge table for the cladding and one for the core.

To get the $x, y$ data from the array, the program splits the data into two sections. The first section includes the fiber edge from $-45^{\circ}$ to $45^{\circ}$ and $135^{\circ}$ to $225^{\circ}$. In this section, the data are scanned horizontally line by line. The edge-finding routine is flexible and can find the edge at a specific fraction of the maximum intensity or at the inflection point. The second section includes the fiber edge from $45^{\circ}$ to $135^{\circ}$ and $225^{\circ}$ to $315^{\circ}$, and the program scans the data in vertical lines.

The program fits an ellipse to the points in the edge table in two stages. The program first uses least median of squares (LMS) to remove outliers and then least sum of squares (LSS) to fit an ellipse to the remaining points. This procedures differs from the procedure used by [3]. 
LMS is a robust method that minimizes the median of the squares of the residuals and is insensitive to outliers [6]. A residual is the distance between a data point and the fitted ellipse, though that distance need not correspond to a physical distance, depending on the fitting algorithm.

The program offers three options for the LSS fit; each uses a different definition of the distance. The first option is called "convenient." It uses a residual which is convenient for calculation. This residual is not the Euclidean distance between the point and the ellipse. The second option, called "perpendicular," uses the perpendicular Euclidean distance between the point and the ellipse. The third option is called "rigorous." Here we assume that the horizontal (x) coordinate of an edge point from a vertically scanned line of data has no uncertainty and define the residuals in the vertical $(y)$ direction. Likewise, we define the residuals of a horizontally scanned line in the vertical direction. We had hoped that the "rigorous" fits would substantially improve the fit. However, for the images we used, the noise was small, and the fits were not significantly different. The difference between the convenient LSS and the perpendicular Euclidean distance LSS is discussed more rigorously in [7].

The LMS stage does not radically improve the fit to an undamaged fiber end. However, if the fiber end is damaged, the LMS stage removes the points at the damaged part of the fiber and still achieves a good fit [3]. The ends of the fibers we used had no noticeable defects in the edge; the fits from the three methods were not significantly different.

In addition to the edge table in Cartesian coordinates, we found it useful to look at the edge in polar coordinates. In a plot of fiber radius as a function of angle (an $r-\theta$ plot), an ellipse appears as a sinusoid with two maxima and two minima. The maxima are the radii along the major axis of the

ellipse. Noncircularity is easier to detect in a plot of this type. The scale can be expanded about the average radius to show fine detail in the edge table.

\section{Defocus}

Our ability to focus the microscope was the largest source of uncertainty in the diameter measurements. The image on the monitor appeared sharp over a focusing range of $2 \mu \mathrm{m}$. We call this range the operating depth of focus to distinguish it from the theoretical depth of focus of the objective alone. We looked at the mean diameter of a fiber end as a function of defocus. Define positive defocus when the fiber is too close to the objective, negative defocus when the fiber is too far from the objective. Best focus is the middle of the operating depth of focus. To find the best focus, we start with the fiber out of focus and move it toward focus until the image stops changing. We call this point the edge of focus. If we approach from too far away, we find the back edge of focus. If we approach from too near, we find the forward edge of focus. We then move the fiber $1 \mu \mathrm{m}$, one half the operating depth of focus, into focus. The fiber is now near the middle of the operating depth of focus. Figure 3 is a graph of mean diameter as a function of defocus for two separate runs. 


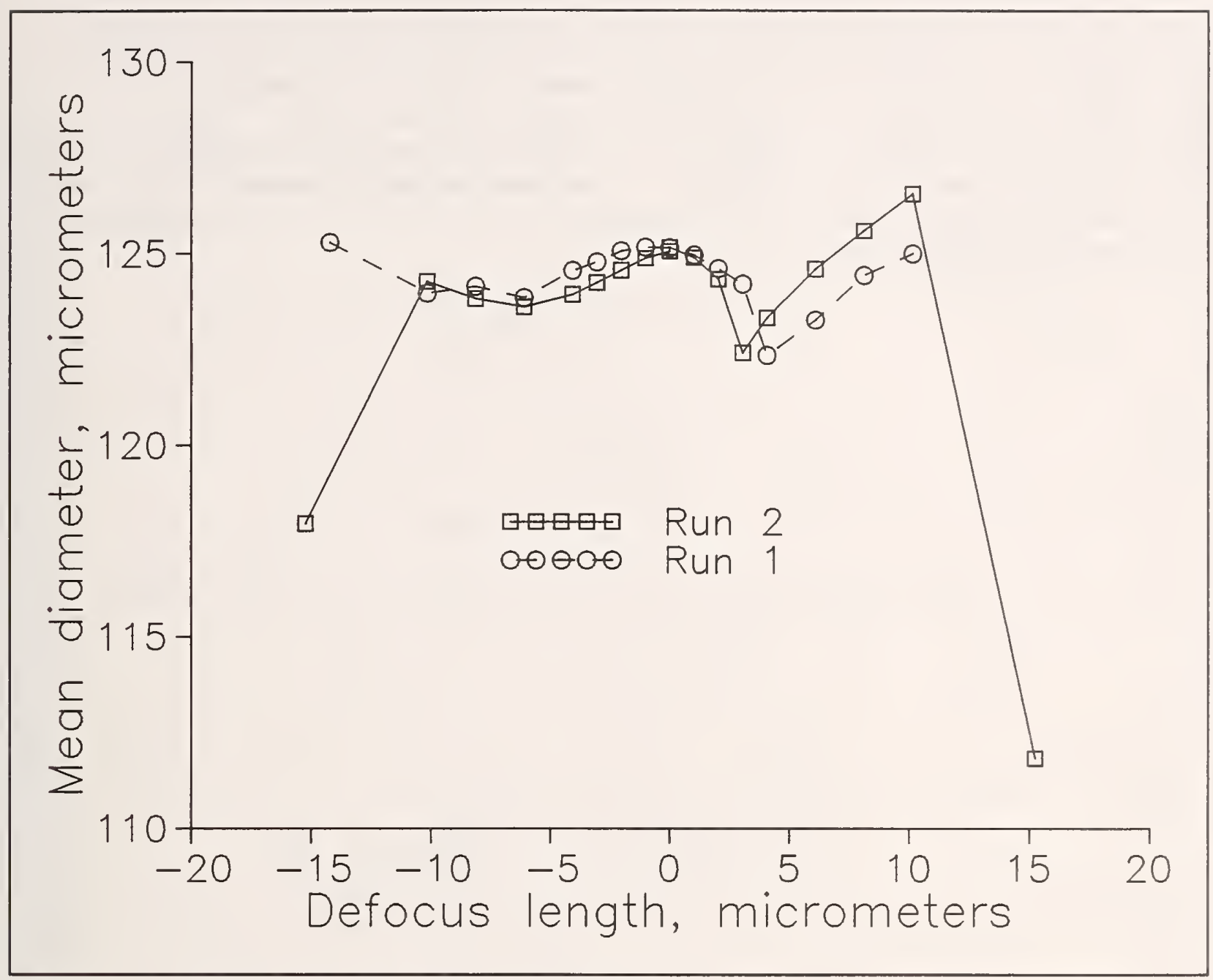

Figure 3. Mean diameter as a function of defocus.

In the first set of data for mean diameter as a function of defocus, we moved the fiber back to the best focus before taking it out of focus for each point (Run 1). In other words, we found the best focus and digitized the image. We moved the fiber $1 \mu \mathrm{m}$ out of focus and digitized another image. Then, we moved the fiber to best focus, moved it $2 \mu \mathrm{m}$ out of focus, and digitized a third image, and so on. To eliminate backlash, we always found the best focus by first finding the back edge of focus.

In Run 2, we first found the back edge of focus and then best focus. We moved the fiber forward, without returning to focus as in Run 1, to acquire positive defocus data. We then found the forward edge of focus and best focus. We moved the fiber away from the objective, without returning to focus, to acquire negative defocus data. Images that had net defocus greater than $2 \mu \mathrm{m}$ were visibly out of focus.

Both sets of data have very similar shapes. The best focus appeared to be at a local maximum of the mean diameter. However, we find the best focus in a subjective manner and cannot resolve the curve accurately enough to state positively that the best focus is a local maximum. The mean diameter has a range of $0.3 \mu \mathrm{m}$ over the operating depth of focus. However, $0.3 \mu \mathrm{m}$ is an overestimation of the uncertainty due to focussing. We can repeatably find the best focus well within the operating deptly of 
focus. We made 12 runs in order to find a statistical estimate of the uncertainty. For each run, we found the best focus starting at the back edge and starting at the front edge. Figure 4 represents the mean diameter for each of the 12 runs. The mean diameter of all of the measurement is $125.122 \mu \mathrm{m}$. The overall spread of values is $0.1 \mu \mathrm{m}$ counting an apparent outlier at Run 7 . Figure 3 shows a maximum in the mean diameter at 0 defocus, so we will assume that the region near best focus is nearly flat. We will also assume that the distribution of values about the mean is Gaussian. Without Run 7 , the standard deviation or $1 \sigma$ uncertainty of the mean diameter is $\pm 0.018 \mu \mathrm{m}$. The $3 \sigma$ uncertainty is $\pm 0.054 \mu \mathrm{m}$, well within the goal of $0.1 \mu \mathrm{m}$.

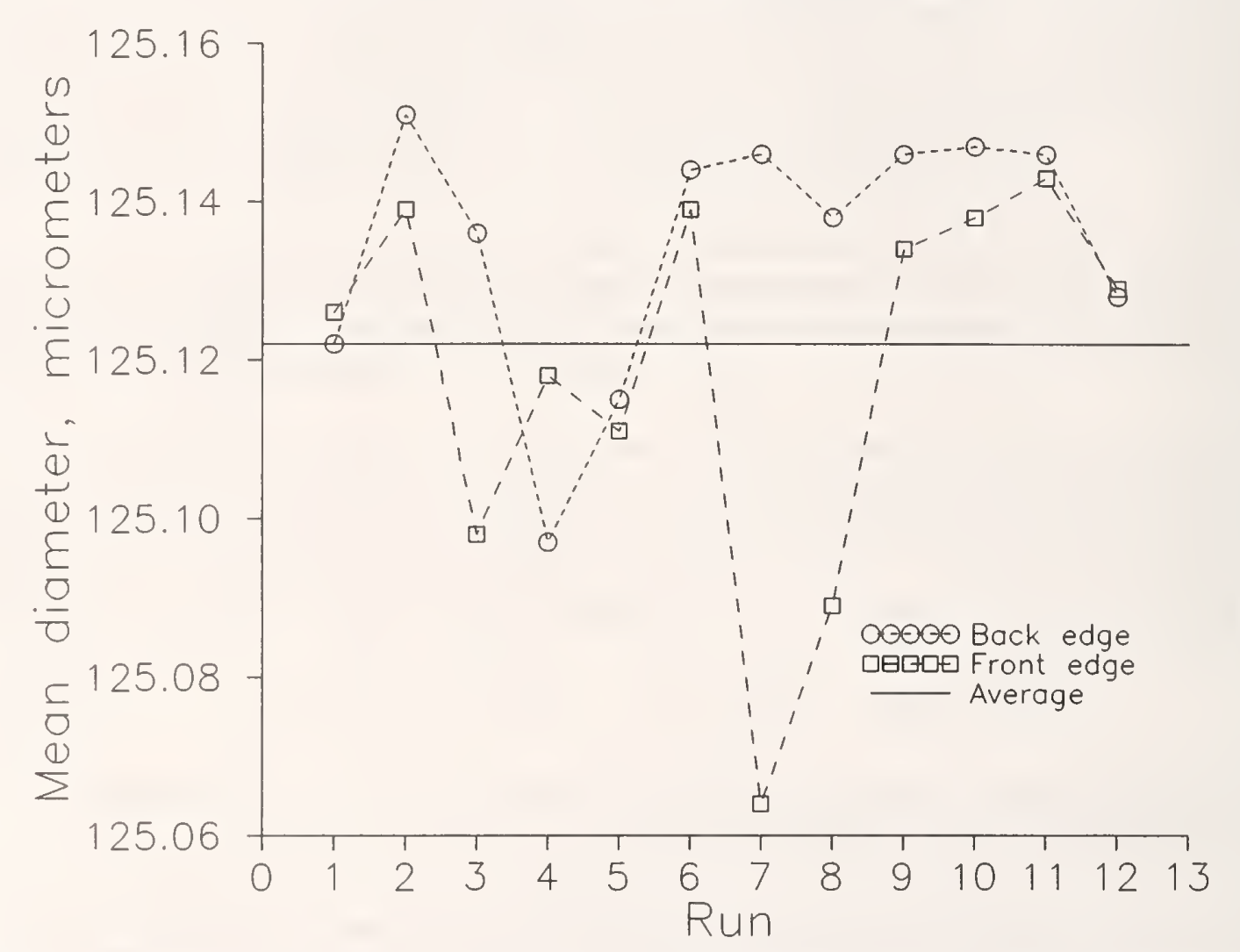

Figure 4. Mean diameter at best focus.

The mean diameter of the fiber is $124.935 \mu \mathrm{m}$ with a $3 \sigma$ uncertainty of $\pm 0.04 \mu \mathrm{m}$ as measured on the contact micrometer. The video microscope therefore displays an error of $0.19 \mu \mathrm{m}$, which exceeds the goal of $0.1 \mu \mathrm{m}$ absolute accuracy. The $1 \sigma$ uncertainty in this error is the standard deviation of the mean of the data, or $\pm 0.004 \mu \mathrm{m}$. The $3 \sigma$ uncertainty of the error is $\pm 0.012 \mu \mathrm{m}$.

Figure 5 shows the noncircularity for each of the runs. The average noncircularity was $0.054 \%$. Neglecting Run 7 and assuming that the distribution about the mean is Gaussian, the $1 \sigma$ uncertainty is $\pm 0.003 \%$. 


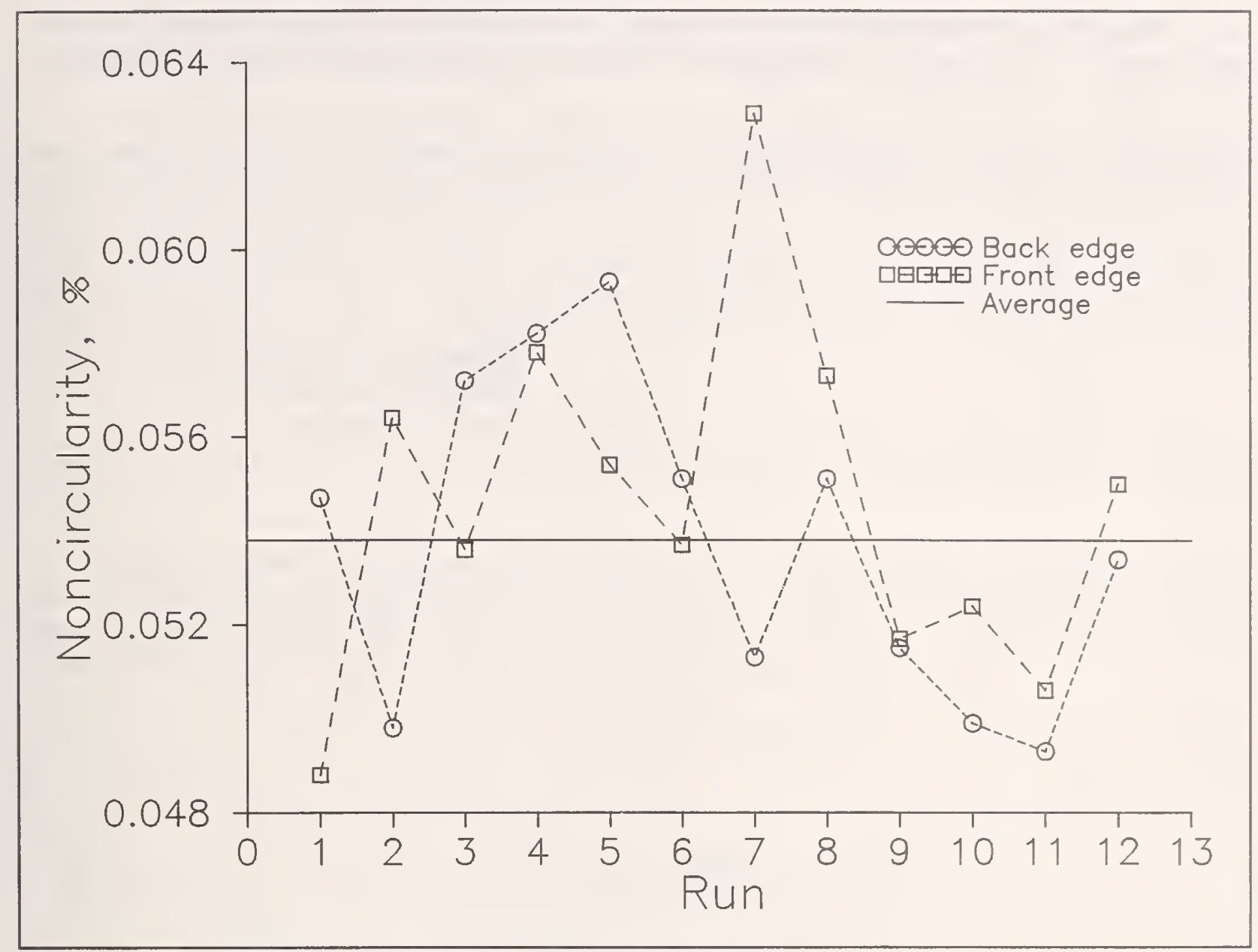

Figure 5. Noncircularity at best focus. 
Figure 6 shows the angle between the major axis and the horizontal plane for each run. The average angle is $-80^{\circ}$ and, by reasoning similar to that for the mean diameter, the $1 \sigma$ uncertainty is $\pm 8^{\circ}$.

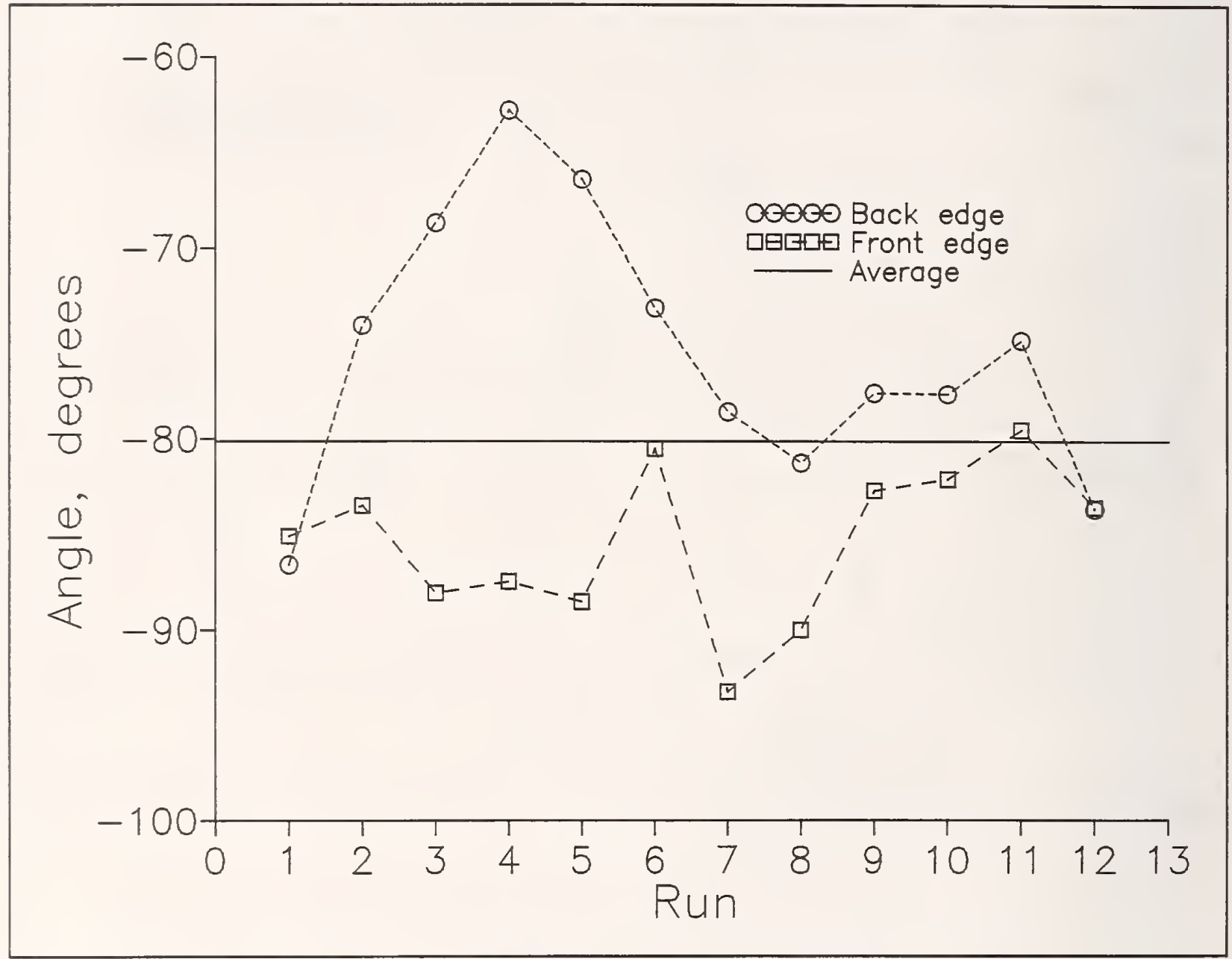

Figure 6. Angle of major axis at best focus.

5. Tilt

When the fiber end is not perpendicular to the optical axis, the data of Figure 3 suggest that the out-of-focus sections of the end will appear too small, whereas the in-focus sections should remain unchanged. The net effect of the tilt, therefore, is a smaller mean diameter, an altered noncircularity, and a shift in the apparent position of the major axis.

Consider a fiber end tilted about some axis. The diameter parallel to that axis will appear unchanged, while the diameter perpendicular to the axis will appear too small. As the tilt angle becomes larger, the apparent difference between the two diameters will increase. Regardless of the true position of the major axis, the apparent major axis will ultimately align with the tilt axis. The noncircularity can follow either of two paths. If the diameter parallel to the tilt axis is the major axis of the fiber end face, the noncircularity will increase with increasing tilt. If, however, that diameter is the minor axis, the noncircularity will first decrease as the apparent perpendicular diameter is brought 
nearer the value of the parallel diameter. Then, once the perpendicular diameter is made smaller than the parallel diameter, the noncircularity will increase once again.

We tested these suppositions by using the angle adjustments on the beam splitter mount. The beam splitter mount has two screws which control two orthogonal rotations. The distance between the screws and their pivots is $32.8 \mathrm{~mm}$ (1.29 in). The screws have 80 threads per inch. Let us say that positive pitch brings the top edge of the fiber away from the objective and that positive yaw brings the right edge of the fiber end away from the objective. When we took data changing yaw, we focused on the top and bottom of the fiber image because these do not change their relative positions when we change the yaw angle. When we changed pitch, we focused on the left and right edges. We always came from the back edge of focus.

In the first set of data, we used a very nearly circular fiber, whose mean diameter is $124.935 \mu \mathrm{m}$, noncircularity is $0.3 \%$, and major axis angle is $-70^{\circ}$. Our predictions are all supported by the data.

Figure 7 is the mean diameter as a function of tilt. As with defocus in Figure 3, the mean diameter appears to have a maximum at $0^{\circ}$ tilt. For the same angle, yaw appears to have a greater effect on the mean diameter than pitch. This could be due to the different pixel geometries in the two directions. (The travel of the screw limited the amount of negative yaw.)

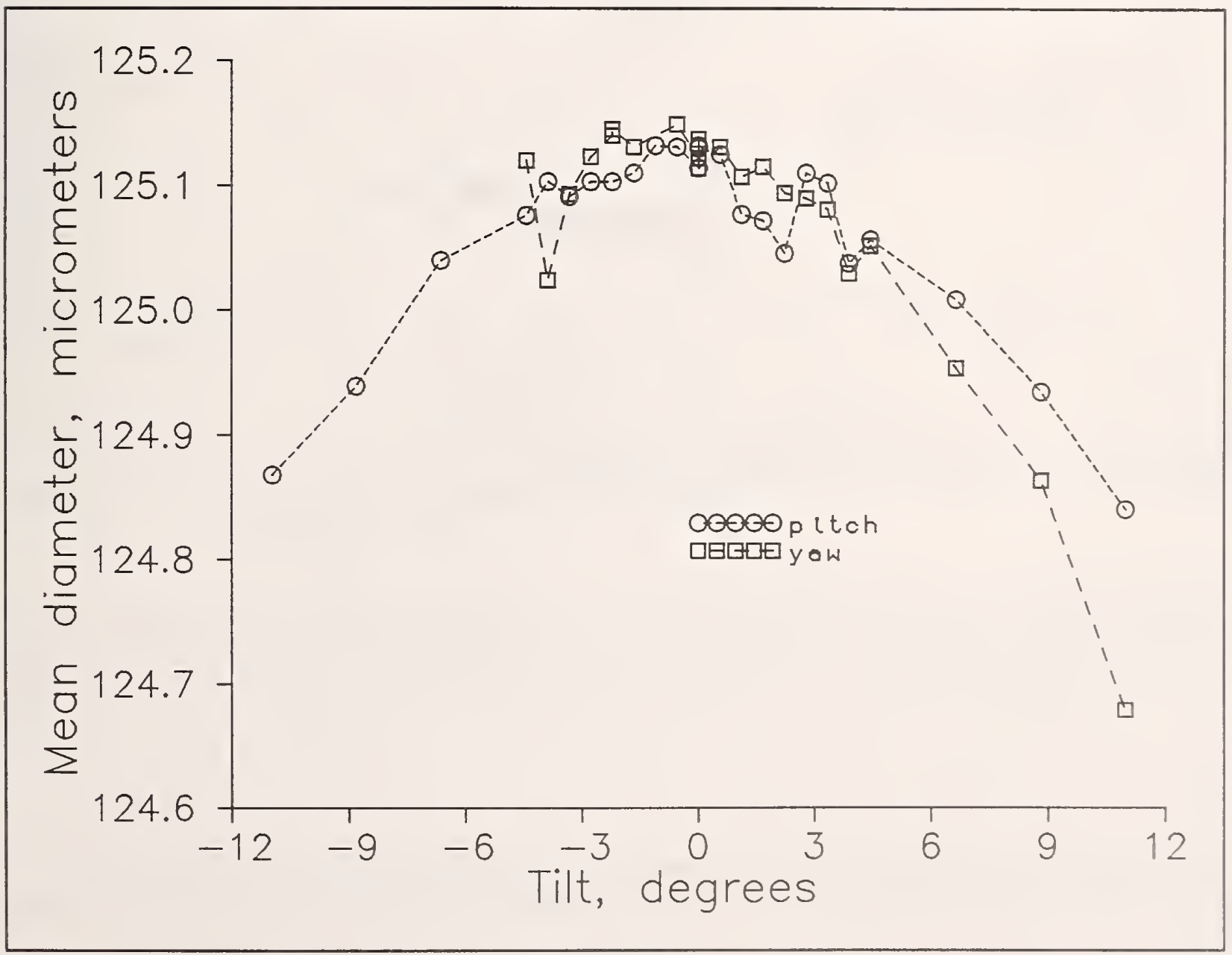

Figure 7. Mean diameter as a function of tilt angle. 
Figure 8 is the noncircularity as a function of tilt angle. The noncircularity appears to have a minimum at $0^{\circ}$ yaw. Because the major axis of the fiber is near $-90^{\circ}$, we expect the noncircularity to decrease at small pitch; in fact, it decreases slightly at $\pm 3^{\circ}$ pitch and then increases. Yaw also appears to have the greater effect on the noncircularity.

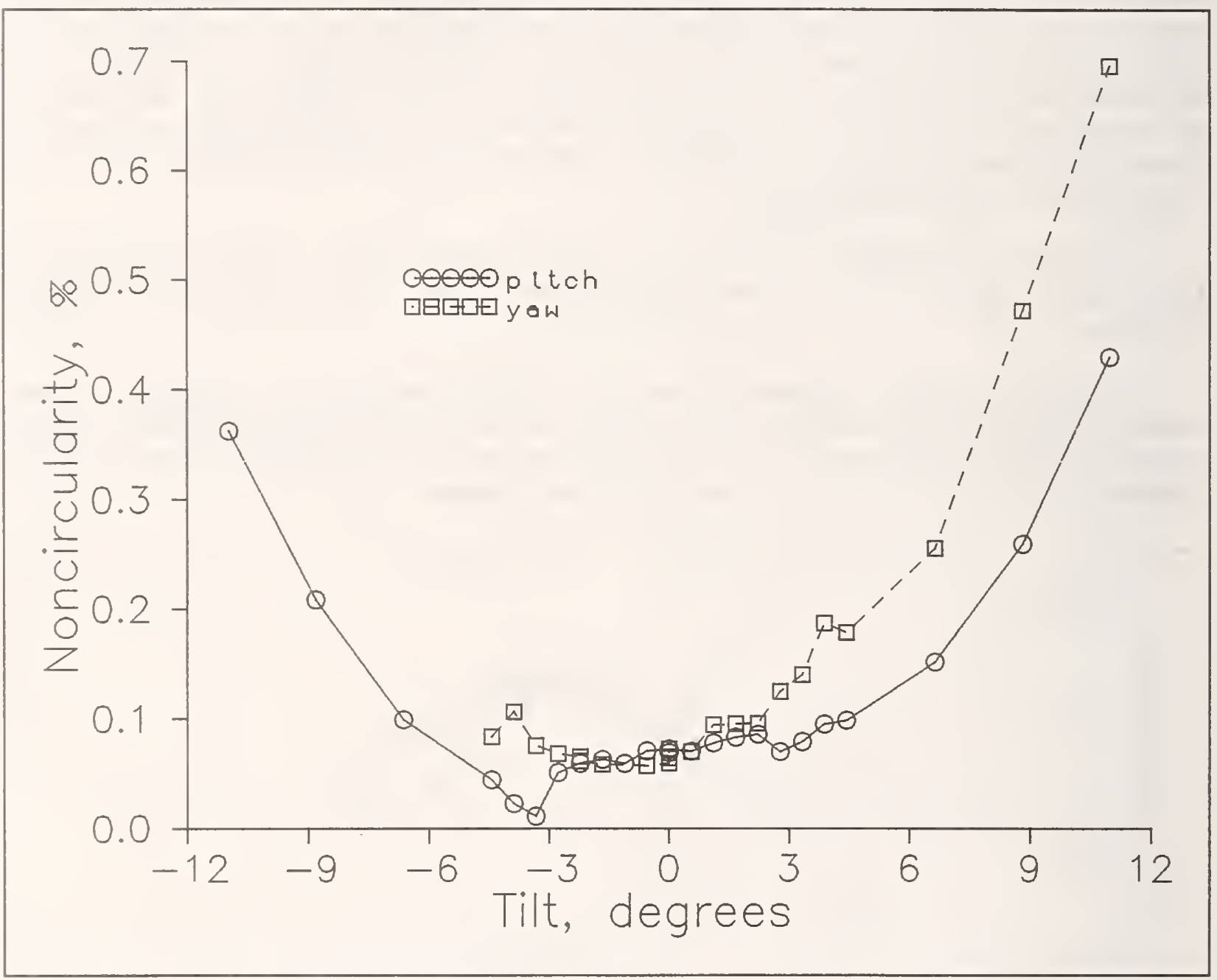

Figure 8. Noncircularity as a function of tilt angle. 
to a period of two pixels. We used a discrete one-dimensional convolution to simulate its effect on the image. We saw no effect on any of the geometric parameters or on the edge table.

We also did a simulation to see whether pixelating changed the location of the edge. The pixels are on average spatial low-pass filters. We used a one-dimensional square convolution kernel to approximate a low-pass filter and convolved it with the theoretical edge profile. The original edge profile and the shifted edge profiles are shown in Figure 22. The position axis is expanded about 0 to make the shift in the intensity at the edge more clear.

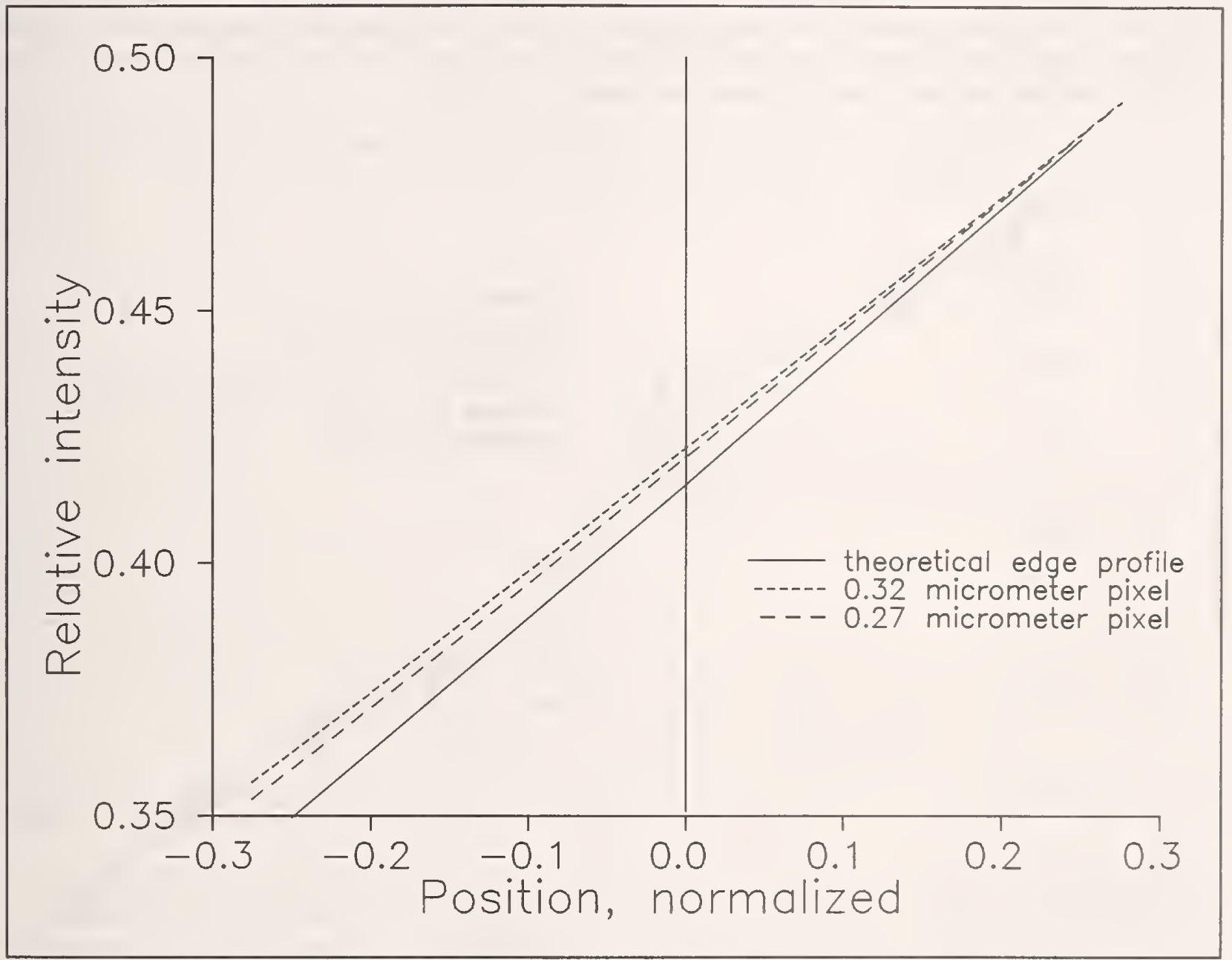

Figure 22. Intensity at the edge: theoretical and pixelated

1 resolution limit $=3.14$.

This simulation indicates that low-pass filtering by the pixels increases the apparent intensity at the edge by 0.005 . The effect of looking at an intensity higher by 0.005 would be a mean diameter error $+0.01 \mu \mathrm{m}$, a value not large enough to account for the apparent discrepancy. However, since the real and theoretical edge profiles are different, this simulation is meaningful only qualitatively.

\section{Fiber Rotation}

The barrel in the SRM holder is free to rotate and is marked at every $45^{\circ}$. Rotating the fiber about its axis is useful to differentiate between the true geometry of the fiber itself and artifacts of the 
microscope. Ideally, the noncircularity and the mean diameter should not change as the fiber is rotated. The angle of the major axis should move by the same angle as the fiber. We used a very nearly circular fiber, noncircularity $0.03 \%$, to investigate the effects of rotating the fiber.

We rotated the fiber in $45^{\circ}$ increments and took an image at each increment. Figure 23 is the angle of the major axis as a function of the angle of fiber rotation. The angle of the major axis moves with fiber rotation but not as we anticipated. When the rotation angle is near $0^{\circ}$, the angle of the major axis is very close to what was measured by the contact micrometer. However, as we rotate the fiber, the measured angle moves away from our expectation. Initially, we thought that this was due to tilt and adjusted the tilt to try to remove the glitch. The data labeled "center" are for tilt angle as close to 0 as possible using the calibration method described before.

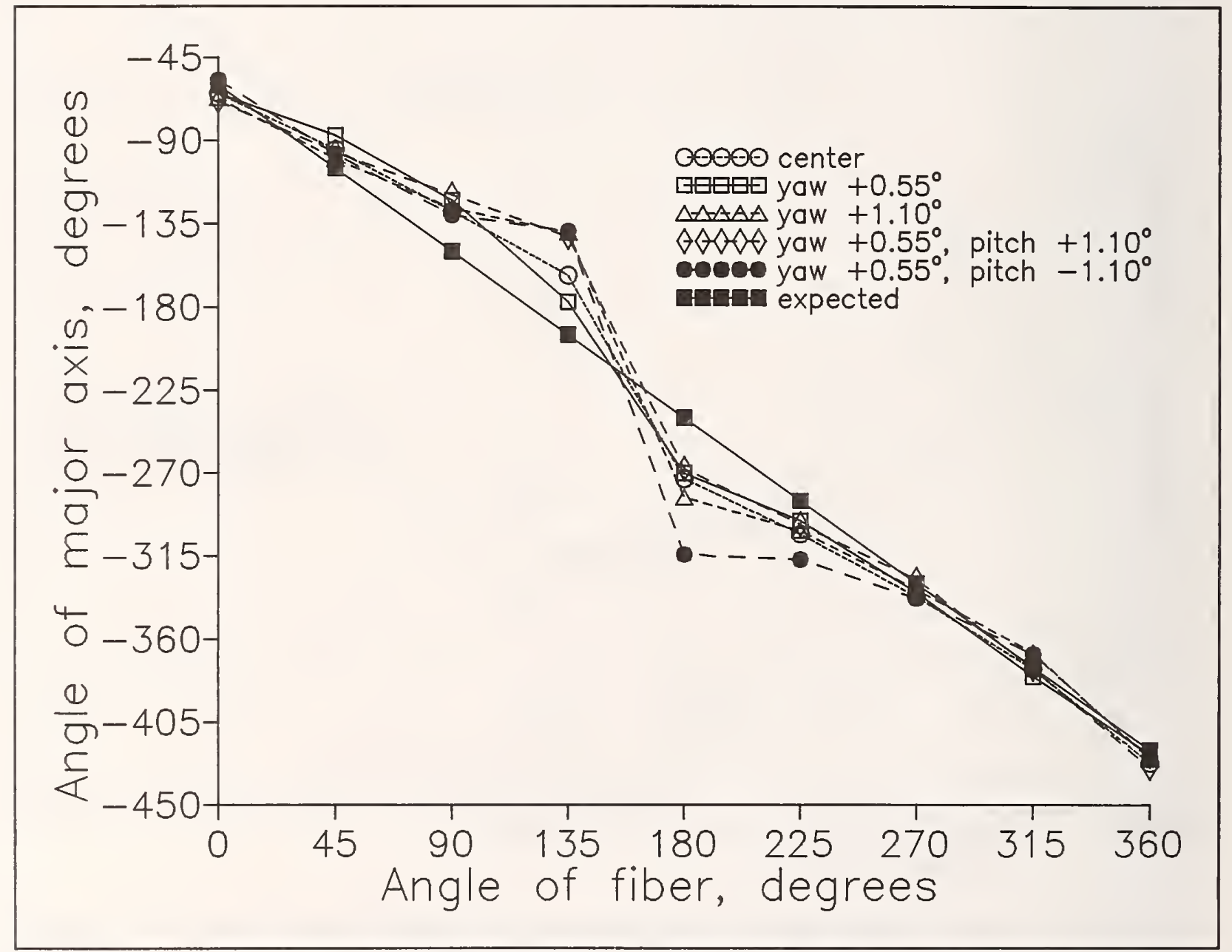

Figure 23. Angle of major axis as a function of fiber rotation angle.

As Figure 23 shows, we could minimize the glitch but not remove it. We think it comes about because the axis of the fiber is not precisely perpendicular to the axis of rotation of the barrel. This is verified by noting that the image of the end moved upward slightly at $0^{\circ}$ and downward at $180^{\circ}$.

Because the fiber is not parallel to the optical axis, the angle between the fiber end and the optical axis changes as the fiber is rotated. Since we minimize tilt with the fiber at $0^{\circ}$, the tilt will not be minimized at $180^{\circ}$. But we can instead minimize the average tilt for all rotations by minimizing the glitch in the curve of major axis angle as a function of fiber rotation angle. The mean diameter as a 
function of the fiber rotation angle, Figure 24, does not display a glitch at $180^{\circ}$; the outlier at $90^{\circ}$ is probably a blunder.

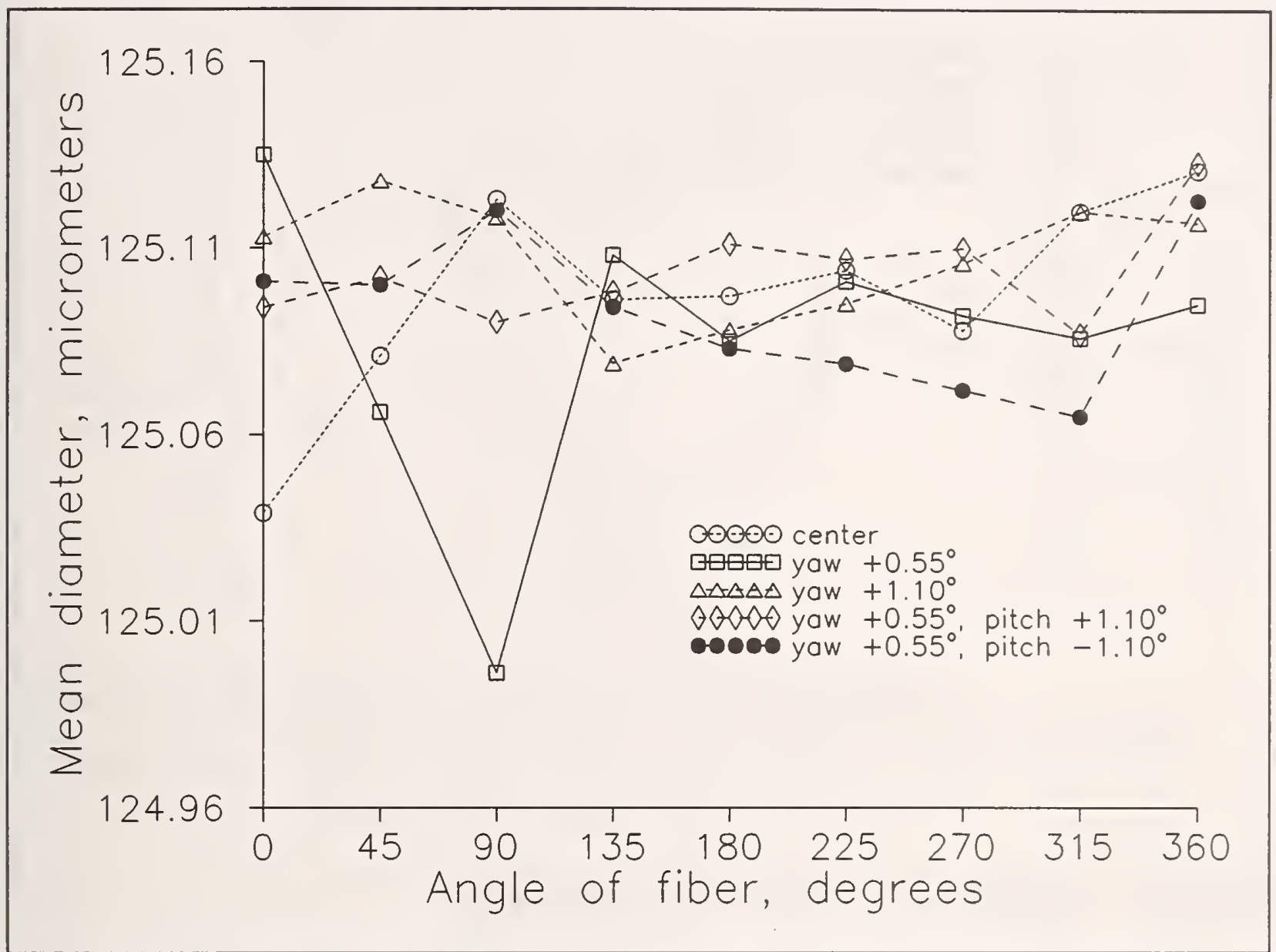

Figure 24. Mean diameter as a function of fiber rotation angle.

Figure 25 is the noncircularity as a function of fiber rotation angle. The noncircularity also changes as the fiber rotates. There appears to be a glitch between $135^{\circ}$ and $225^{\circ}$. However, the uncertainties due to the cleave and tilt are large enough that most of the data are within each other's uncertainties. 


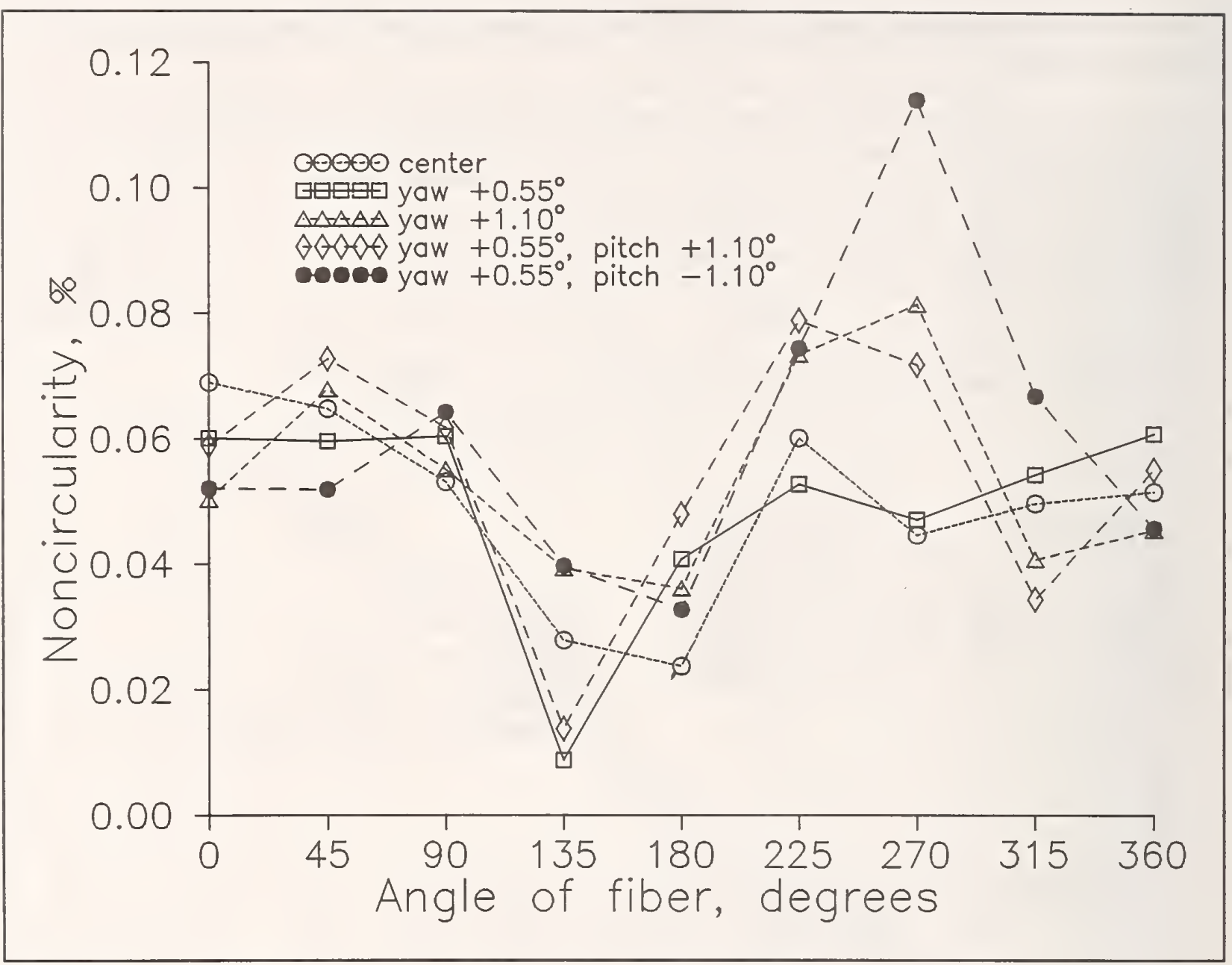

Figure 25. Noncircularity as a function of fiber rotation angle.

\section{Concentricity Error}

Another important parameter is the distance between the center of the cladding and the center of the core. This is also known as the concentricity error. We looked at the vector between the cladding center and the core center as a function of the rotation angle of the fiber. As we rotate the fiber, the magnitude of the vector should not change, but its angle should change by the angle of rotation. That is, the tip of each vector should lie on a circle centered about the center of the cladding. Unfortunately, the magnitude of the vector changes measurably, and the angle does not change by the expected value. Figures 26,27 , and 28 show the tip of the vector that starts at the cladding center $(0$, 0 ) and ends at the core center for three sets of rotation data. 


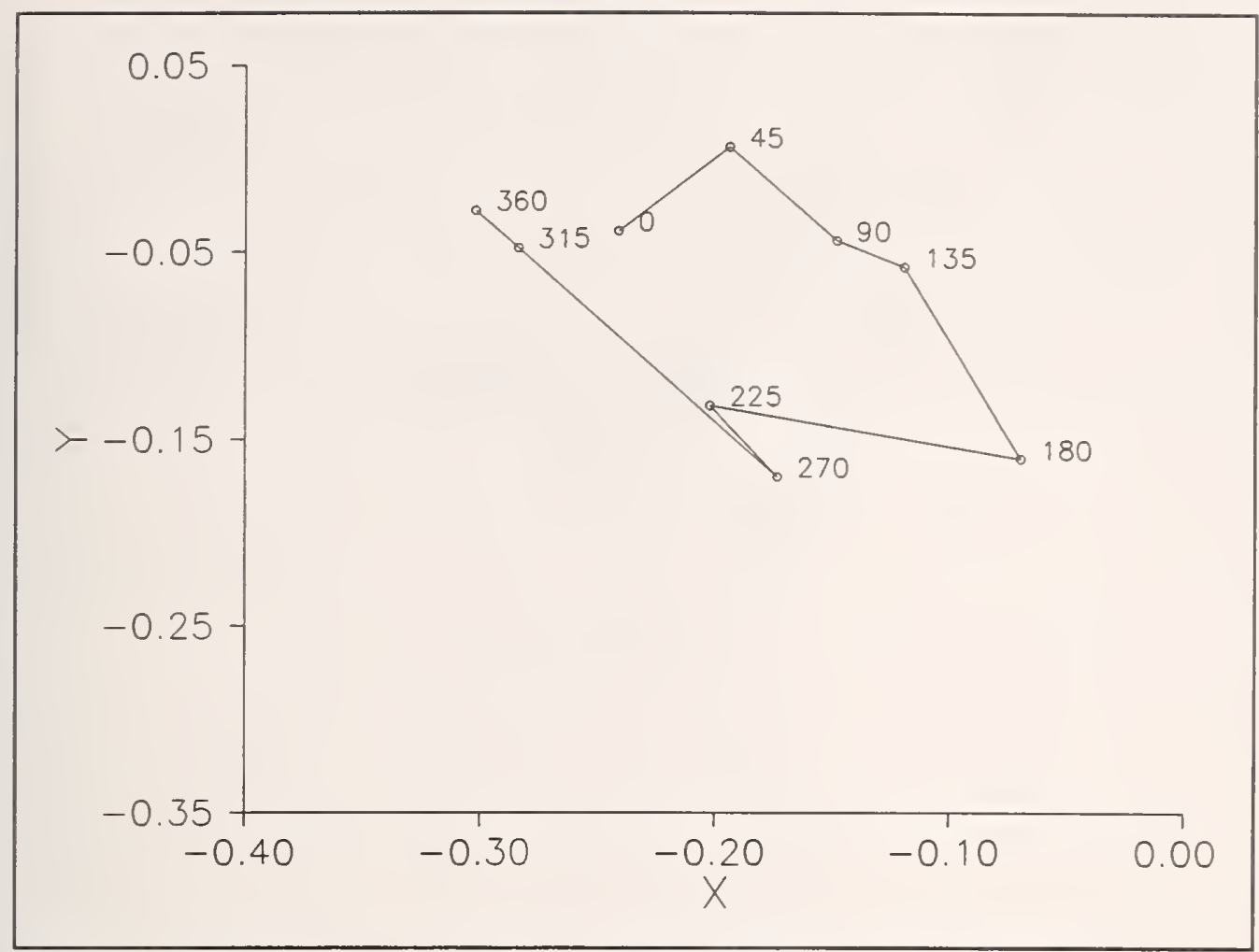

Figure 26. Cladding-core vector as a function of fiber rotation angle (run 1).

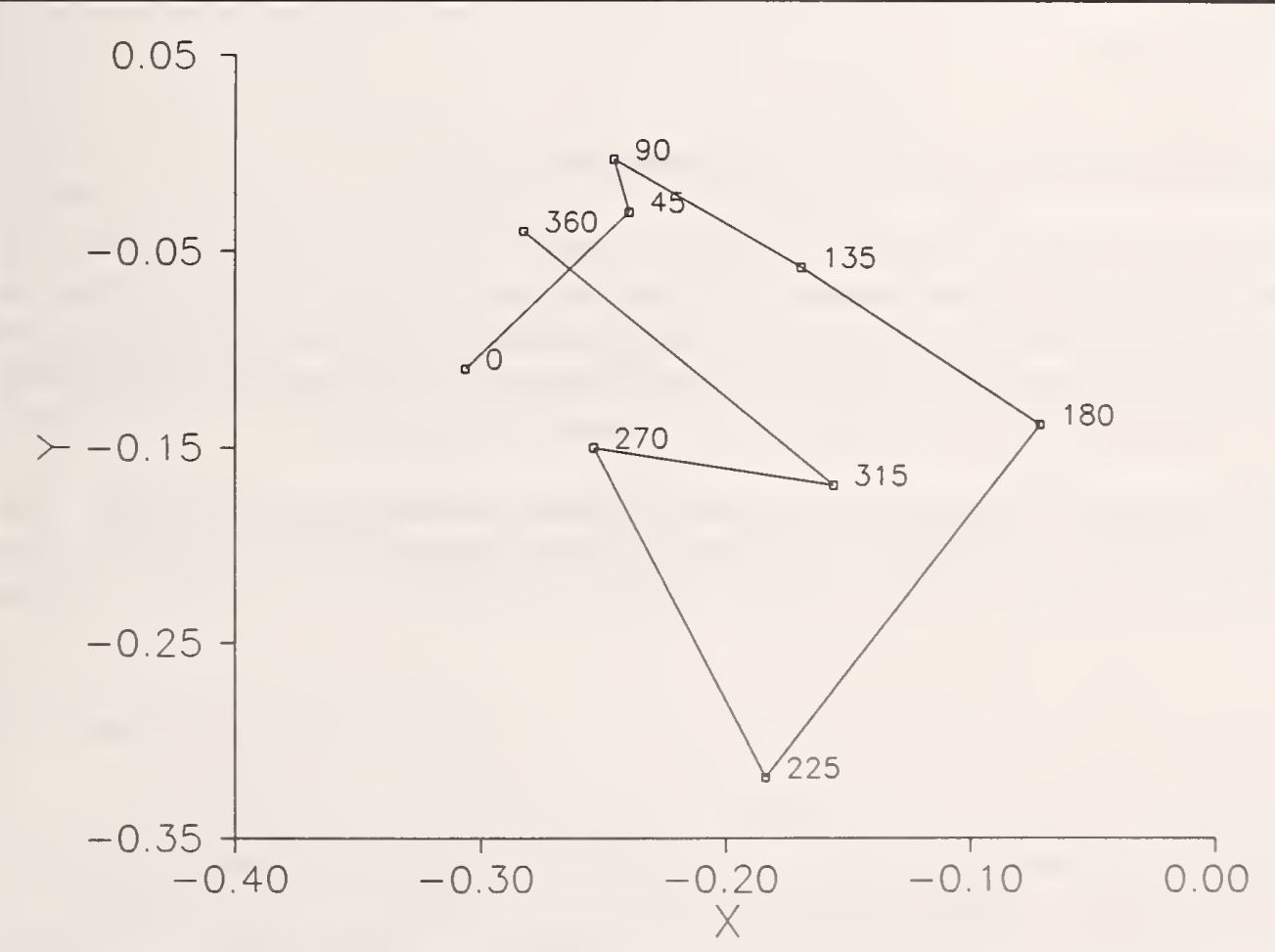

Figure 27. Cladding-core vector as a function of fiber rotation angle (run 2). 


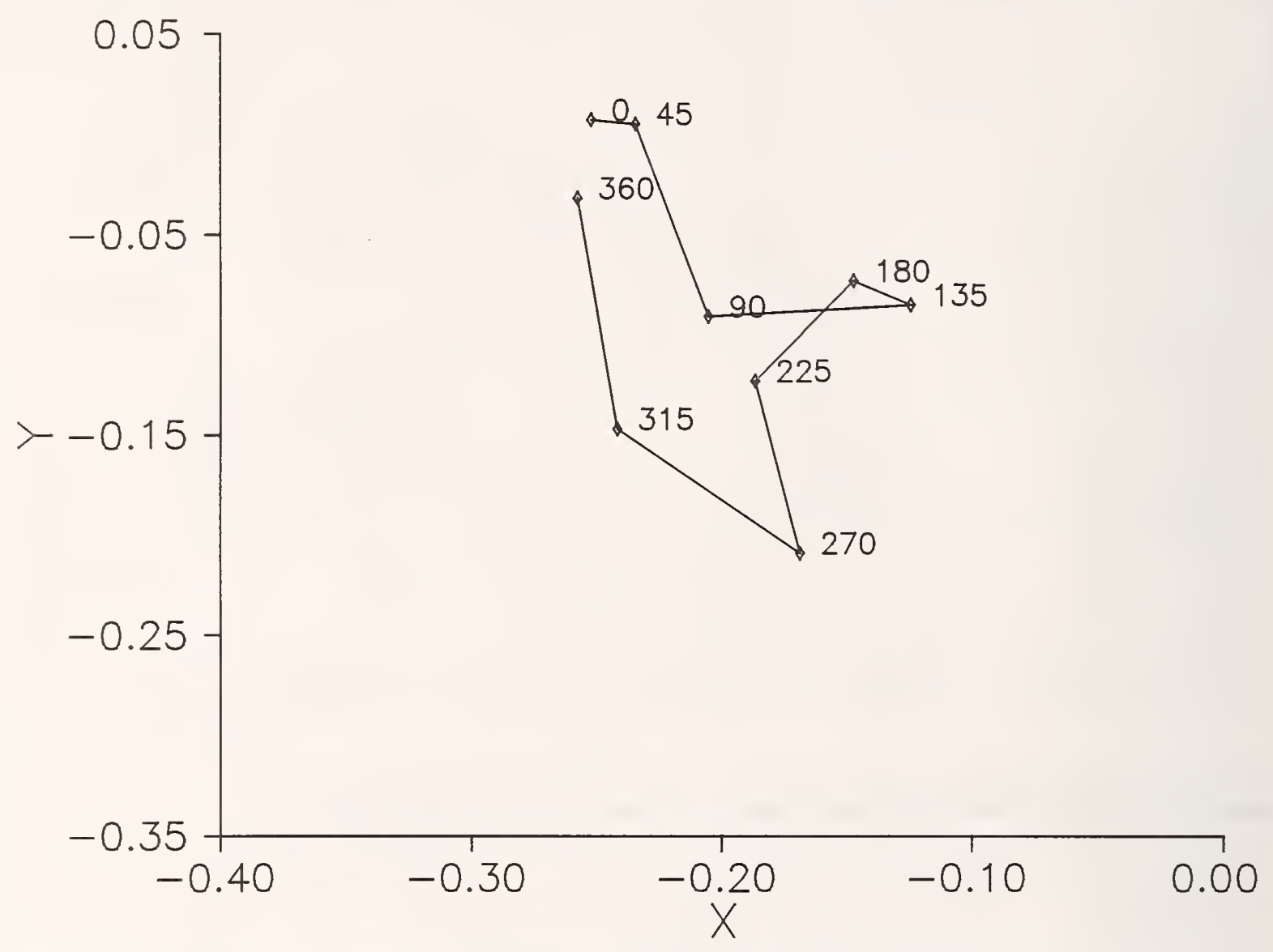

Figure 28. Cladding-core vector as a function of fiber rotation angle (run 3).

In these figures, the data labels represent the angle of rotation of the fiber with respect to an arbitrary starting point. The origin of coordinates is the center of the cladding as calculated from an ellipse fit. In each case, the vector rotates only very approximately in a circle, but the center of rotation is a point removed from the origin by a vector whose magnitude is approximately $0.26 \mu \mathrm{m}$ and whose angle is $-157^{\circ}$. We have been unable to identify the source of this error.

To estimate the uncertainty of the vector, we used the same data as for Figures 4, 5, and 6 . The tips of the concentricity error vectors for these data are plotted in Figure 29. 


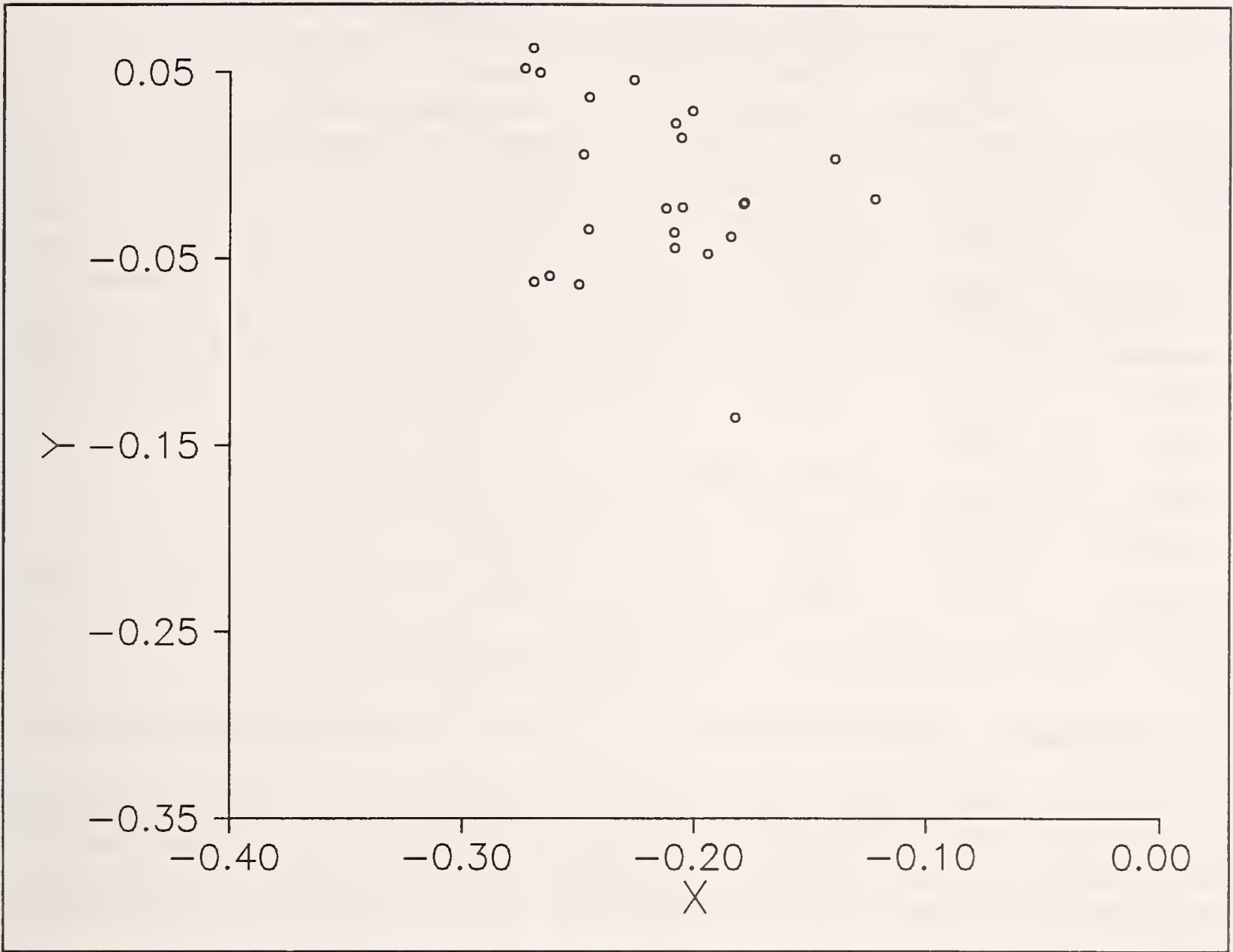

.Figure 29. Core-cladding vectors for data sets of Figures 4, 5, 6.

We found the center of the data points in Figure 29 and the distance between the center and each point. The standard deviation of these distances is an estimator of the uncertainty of the magnitude of the vector; it is equal to $0.057 \mu \mathrm{m}$. The largest change in the vector angle would occur if the $0.057 \mu \mathrm{m}$ deviation were perpendicular to the offset vector. This creates a $1 \sigma$ angle uncertainty of $12^{\circ}$

We have not investigated further, but presumably to calculate the concentricity error accurately, we would have to refer the measurement to the tip of the vector rather than to the center of the cladding. Something along these lines is the procedure actually used in commercial instruments.

\section{Summary of Uncertainties}

Table 1 contains a summary of the uncertainties and their sources. These uncertainties are for single measurements only. The artifact uncertainty comes about because we used an SRM to calibrate the microscope and to calculate the error of the mean diameter. The micrometer measurement contributes a $1 \sigma$ uncertainty of $\pm 0.013 \mu \mathrm{m}$ to the mean diameter measurement. 
Table 1. Summary of $1 \sigma$ uncertainties.

\begin{tabular}{|c|c|c|c|c|c|}
\hline & $\begin{array}{l}\text { Mean } \\
\text { diameter, } \\
\mu \mathrm{m}\end{array}$ & $\begin{array}{l}\text { Non- } \\
\text { circularity, } \\
\%\end{array}$ & ${ }_{0}^{\text {Angle, }}$ & $\begin{array}{l}\text { Vector } \\
\text { magnitude, } \\
\mu \mathrm{m}\end{array}$ & $\begin{array}{l}\text { Vector } \\
\text { angle, }\end{array}$ \\
\hline Focus & 0.018 & 0.003 & 8 & 0.057 & 12 \\
\hline Tilt & 0.0007 & 0.007 & 1.7 & & \\
\hline Cleave angle & 0.0007 & 0.007 & 1.7 & & \\
\hline $\begin{array}{l}\text { Edge-finding } \\
\text { intensity }\end{array}$ & 0.0017 & 0.00007 & 0.07 & & \\
\hline Offset & 0.004 & & & & \\
\hline Artifact & 0.013 & & & & \\
\hline Total $(1 \sigma)$ & 0.023 & 0.01 & 8.4 & 0.057 & 12 \\
\hline $\begin{array}{l}\text { Expanded } \\
(3 \sigma)\end{array}$ & 0.07 & 0.03 & 25 & 0.171 & 36 \\
\hline
\end{tabular}

\section{Other Fibers}

We used a fiber of mean diameter $124.935 \mu \mathrm{m}$ and noncircularity $0.03 \%$ to calibrate the microscope and investigate the uncertainties. We made measurements on a second fiber at each $45^{\circ}$ rotation increment to check the results. The second fiber had a mean diameter of $125.522 \mu \mathrm{m}$, a noncircularity of $0.25 \%$, and a major axis angle of $-89^{\circ}$. This is the same fiber that we used for the second run of tilt data.

Figure 30 is a graph of mean diameter as a function of fiber rotation angle. The mean diameter has been adjusted by subtracting the $0.19 \mu \mathrm{m}$ offset. The measured values are consistently below the micrometer measurement but mostly within the $3 \sigma$ uncertainties of our measurements. 
incoherent source has an infinite coherence parameter. The mutual coherence function of a nearly monochromatic, circular source in a plane a finite distance away can be found using the van CittertZernike theorem.

We used a half-plane, opaque for $y<0$, to simulate the edge of the fiber (in our system, the fiber is back-lighted and appears as a dark circle). In order to simplify interpretation, we normalized all spatial variables by expressing them in units of the resolution limit of the microscope in the object plane. The resolution limit in the image plane is the resolution limit in the object plane times the magnification of the microscope. Two objects separated by one resolution limit in the object plane will be separated by one resolution limit in the image plane. This automatically takes into account the magnification of the microscope. It also makes the coherence parameter an explicit variable in the integral. We also normalized the intensity by dividing it by the intensity at the origin in the absence of the half-plane. The intensity in the image plane is

$$
I(\bar{a}, \bar{b})=C \int_{-\infty}^{\infty} d \bar{x} \int_{0}^{\infty} d \bar{y} \int_{-\infty}^{\infty} d \bar{w} \int_{0}^{\infty} d \bar{z} \operatorname{somb}\left(\alpha r_{1}\right) \operatorname{somb}\left(r_{2}\right) \operatorname{somb}\left(r_{3}\right)
$$

where $C$ is a normalization constant and the sombrero function [12] is

$$
\operatorname{somb}(r)=\frac{2 J_{1}(r)}{r}
$$

$\mathrm{J}_{1}$ is the first-order Bessel function. The arguments of the sombrero functions are the distances

$$
\begin{aligned}
& r_{1}=\sqrt{(\bar{x}-\bar{w})^{2}+(\bar{y}-\bar{z})^{2}} \\
& r_{2}=\sqrt{(\bar{x}-\bar{a})^{2}+(\bar{y}-\bar{b})^{2}} \\
& r_{3}=\sqrt{(\bar{w}-\bar{a})^{2}+(\bar{z}-\bar{b})^{2}}
\end{aligned}
$$

where $r_{1}$ is the distance between two points in the object plane, $r_{2}$ is the distance between the first point in the object plane and the point in the image plane, and $r_{3}$ is the distance between the second point in the object plane and the point in the image plane.

The first sombrero function is the result of the of the Van Cittert-Zernike theorem. The second and third sombrero functions are both the Green's function of the microscope. We normalize Equation (6) by dividing by

$$
\begin{gathered}
N(\bar{a}, \bar{b})=C \int_{-\infty}^{\infty} d \bar{x} \int_{-\infty}^{\infty} d \bar{y} \int_{-\infty}^{\infty} d \bar{w} \int_{-\infty}^{\infty} d \bar{z} \operatorname{somb}\left(\alpha r_{1}\right) \operatorname{som} b\left(r_{2}\right) \operatorname{somb}\left(r_{3}\right), \\
\bar{a}=\bar{b}=0
\end{gathered}
$$

which is the intensity at the origin in the absence of the half-plane. The result,

$$
I_{n o r}=\frac{I(\bar{a}, \bar{b})}{N(0,0)}
$$

is a function that asymptotically approaches 0 as $y$ approaches $-\infty$ and approaches 1 as $y$ approaches $+\infty$. 
The resulting integrals cannot be solved analytically. We evaluated these integrals numerically for several coherence parameters and computed the edge profiles, intensity as a function of distance along the $y$ axis, for several coherence parameters (see Appendix).

Figure 15 shows edge profiles calculated for a number of coherence parameters.

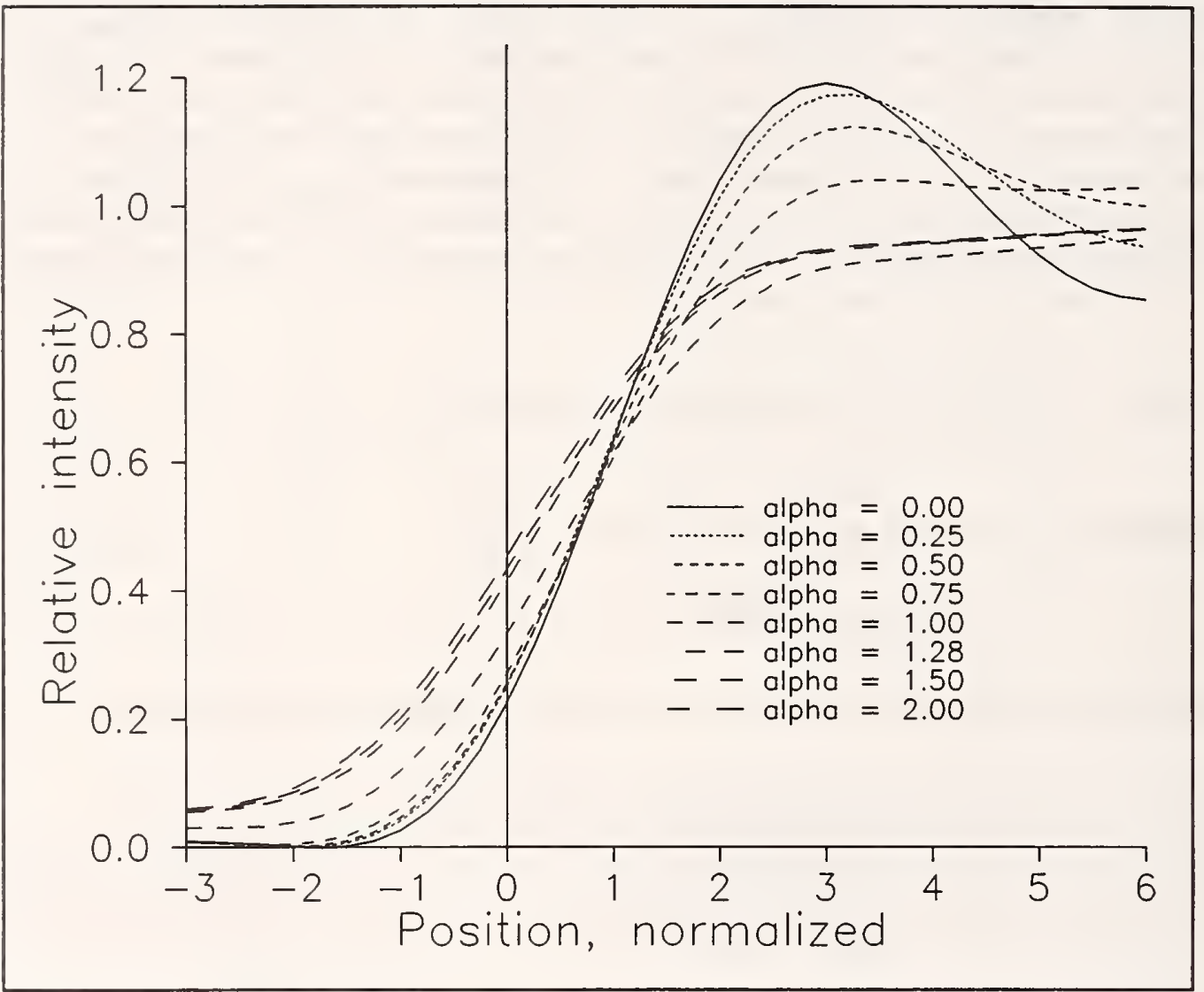

Figure 15. Edge profiles

1 resolution limit $=3.14$.

We also compiled a table of the edge-finding intensities for several coherence parameters. Figure 16 is a graph of those data. We can interpolate for any value of the coherence parameter between 0 and 2 . The edge intensity starts at 0.25 for coherent light, $\alpha=0$, and approaches 0.5 for incoherent light, $\alpha=\infty$. 


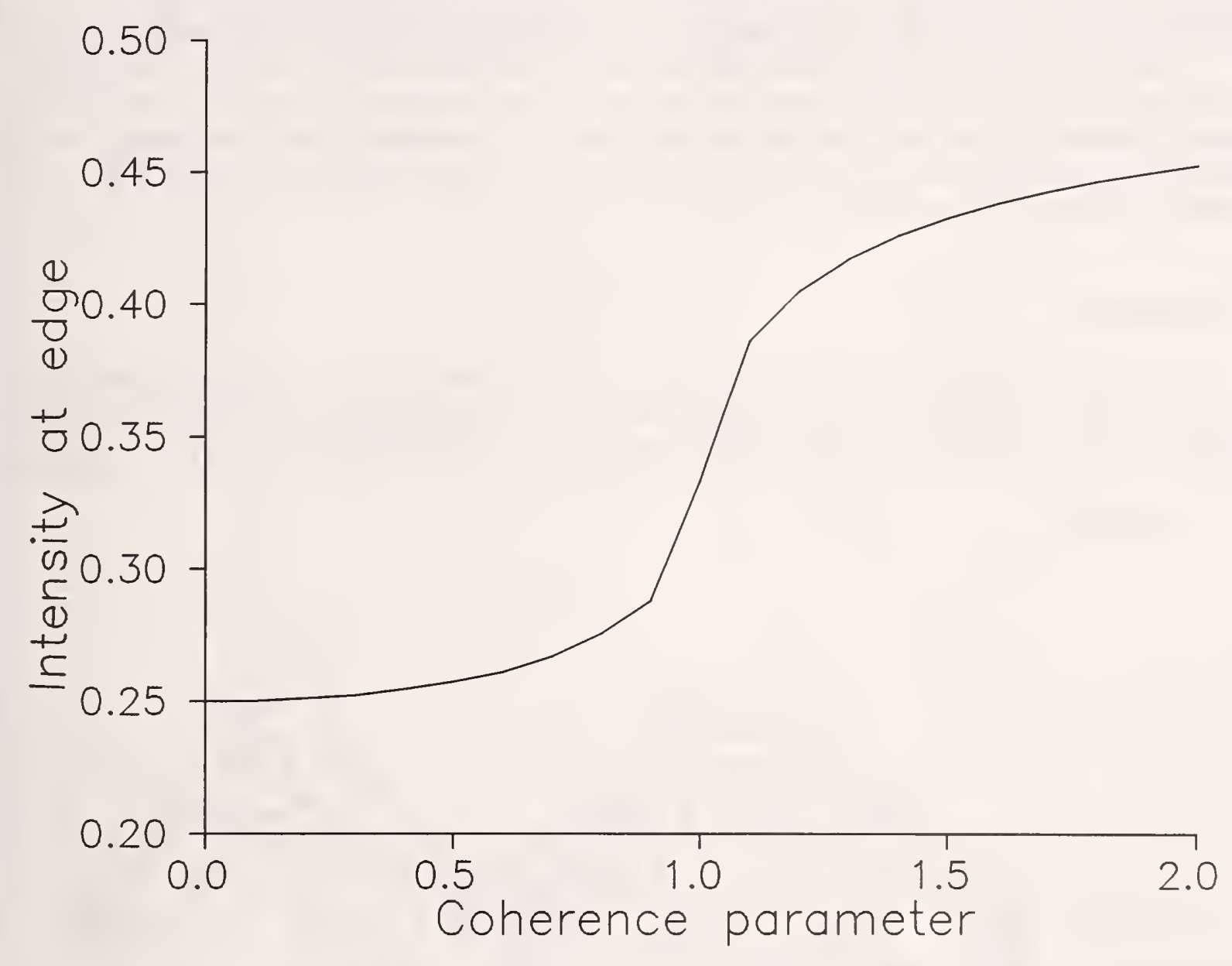

Figure 16. Edge intensity as a function of coherence parameter.

Because the edge-finding algorithm in the computer program uses a relative intensity, we need to know the numerical aperture of the illumination spot. We know the dimension of the spot of light, but we need to measure the distance from the end of the fiber to the metal plate. We did this by using the micrometer for coarse focus. We retracted the fiber into the SRM box, moved the white plate until it touched the cover slip, and noted the position on the micrometer. We then backed off, moved the fiber out of the SRM box, focused on it, and noted the position. The difference between the two positions is the distance between the spot and the objective. We need to find the distance to the focal point of the objective, however, not to the surface of the lens. Since the working distance (the distance between the front surface of the lens and the focal point) of the objective is $0.6 \mathrm{~mm}$, we subtracted $0.6 \mathrm{~mm}$ to find the distance between the spot and the fiber end, or $5.47 \mathrm{~mm}$. When we moved the plate to the cover slip, it met a gradual resistance, not a sudden stop. We estimate that the point at which the plate just touches the cover slip is within $0.25 \mathrm{~mm}$; this is the estimated $3 \sigma$ uncertainty.

Using the $3 \sigma$ uncertainty of the distance between the spot and the fiber end, we estimated the $3 \sigma$ uncertainty in the mean diameter, noncircularity, and major axis angle. We then divided by 3 to estimate the $1 \sigma$ uncertainty of the coherence parameter: the coherence parameter is 1.27 with a $3 \sigma$ uncertainty of \pm 0.02 because of the uncertainty in the fiber-to-spot distance. Using the value 1.27. we estimate the theoretical edge-finding intensity as 0.414 . We estimated the uncertainty of the edge- 
finding intensity by calculating the edge intensity for coherence parameters of 1.25 and 1.29 ; these differ from 1.27 by \pm 0.02 . We thus estimate the $3 \sigma$ uncertainty of the edge-finding intensity as \pm 0.02 .

We did this calculation before taking any data for this paper, and we used the 0.414 intensity for finding all edges. The $3 \sigma$ uncertainty in the mean diameter corresponding to the $3 \sigma$ uncertainty in the edge intensity is $5 \mathrm{~nm}$ and is insignificant compared to the uncertainty due to defocusing. The $1 \sigma$ uncertainty in the edge is $1.7 \mathrm{~nm}$. The $1 \sigma$ uncertainty in the noncircularity is $\pm 0.00007 \%$; the $1 \sigma$ uncertainty in the major axis angle is $\pm 0.07^{\circ}$.

\section{Simulations}

We used an edge profile from Figure 16 to run a simulation of how the pixels in the camera affect the image. While we were looking at plots of the polar edge tables, we noticed that certain aspects of the edge table did not shift in angle as the fiber was rotated. Figure 17 is a polar edge table.

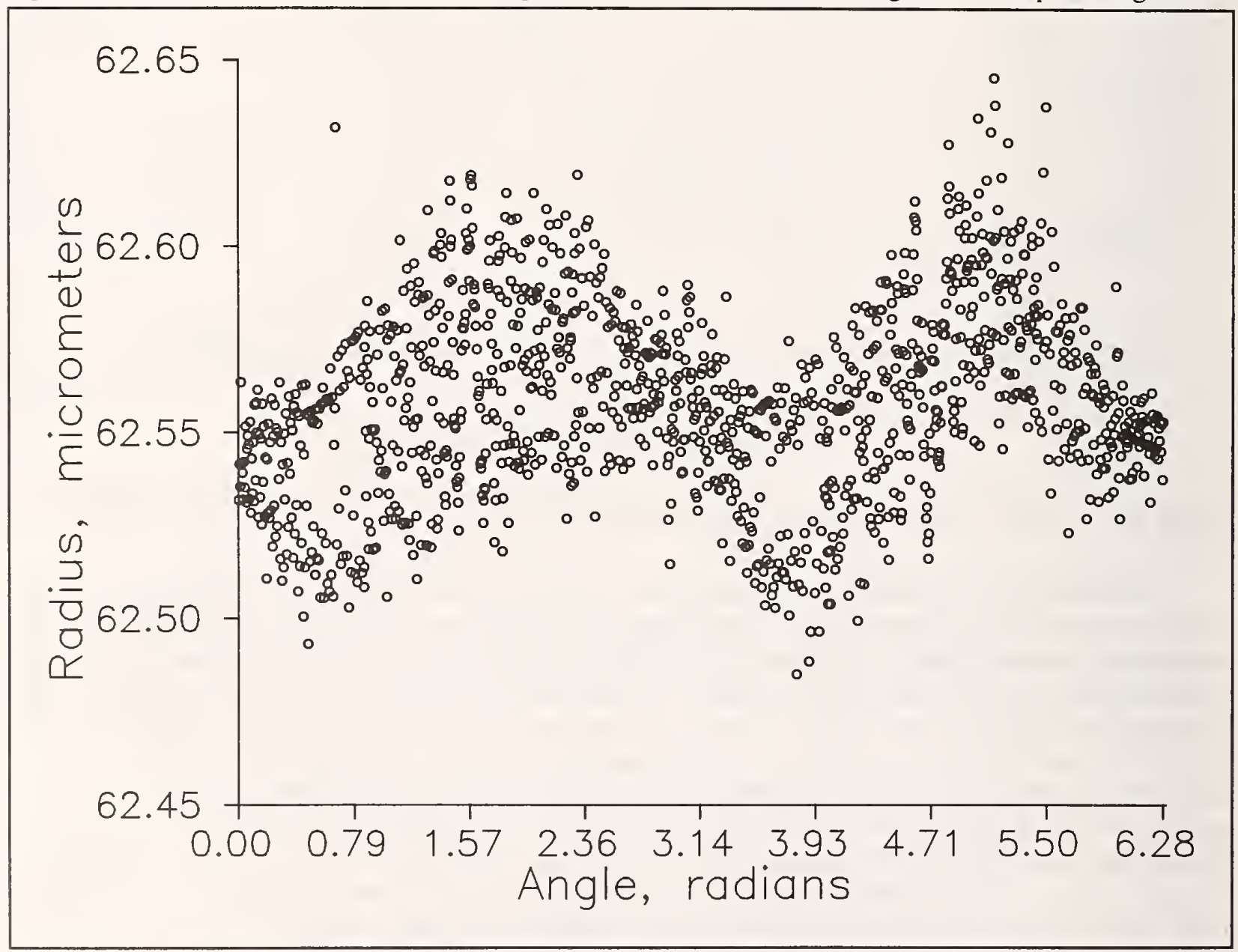

Figure 17. Edge table of a highly circular fiber.

The edge table data display an approximately sinusoidal behavior as well as what appears to be random noise. When the fiber is rotated, the maxima and minima of the distribution change position; this indicates that they result from the noncircularity or cleave angle of the fiber. 
Figure 18 shows the same data as in Figure 17 but expanded around $1.57 \mathrm{rad}\left(90^{\circ}\right)$. On this scale, the data display nonrandom oscillations at $1.57 \mathrm{rad}$ and $4.71 \mathrm{rad}\left(270^{\circ}\right)$. These nonrandom oscillations do not change position when the fiber is rotated, so they are not related to the geometry of the fiber.

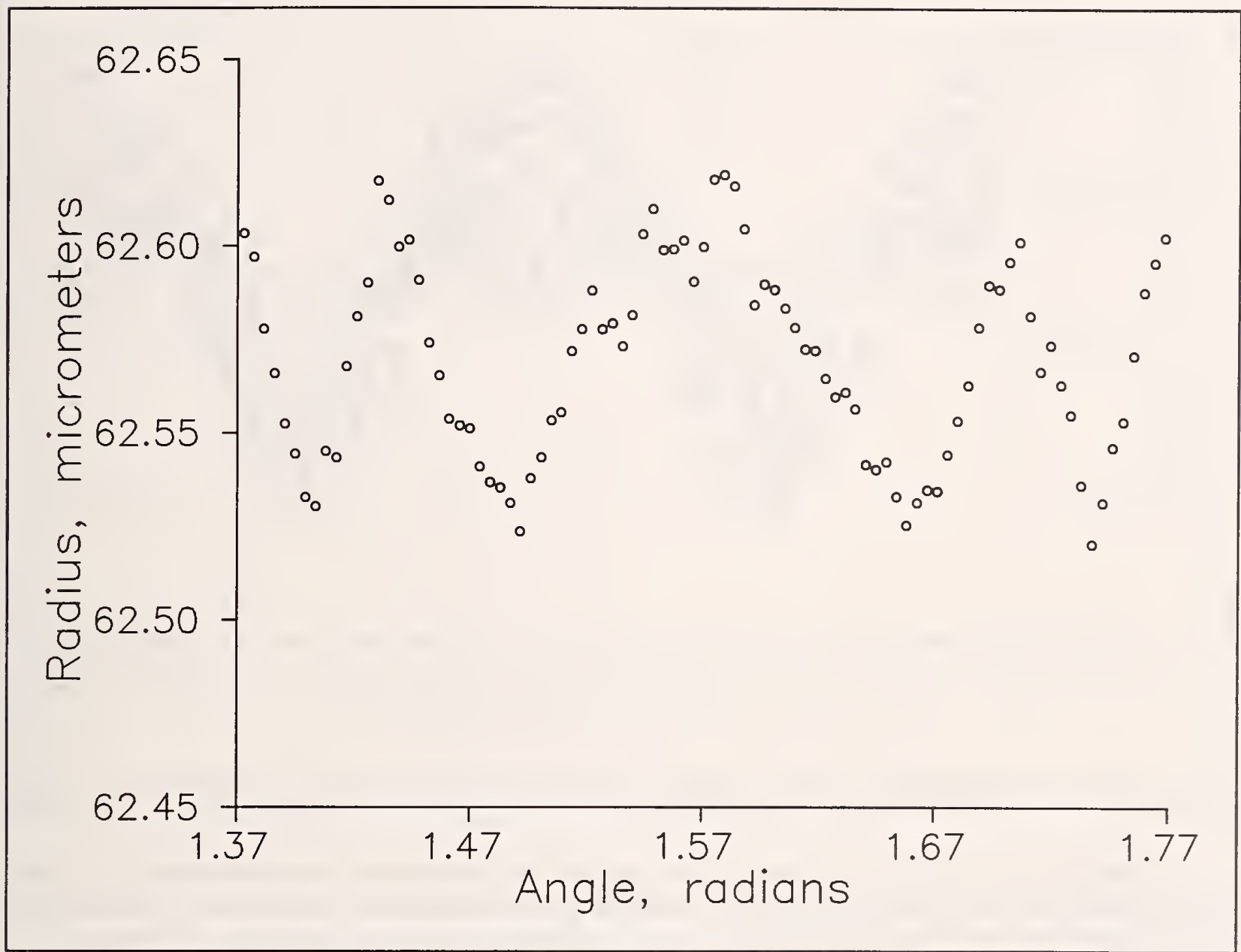

Figure 18. Oscillation of the image of the fiber edge .

Since these nonrandom areas do not move with the fiber, they must be some artifact of the microscope. We used the theoretical edge profile data for a coherence parameter of 1.28 to simulate the effect of finite, rectangular pixels on the image of a circular fiber. We approximated the continuous two-dimensional edge profile of the fiber as a very fine grid of discrete points. The intensity of each point was calculated from an edge profile based on the distance of the point from the center of the fiber. We approximated the pixels as rectangular regions of uniform responsivity. The intensity recorded by one of the simulated pixels is the sum of the values at the discrete points divided by the number of points within the pixel. Since the fiber edge curves only negligibly over a distance equal to the resolution limit of the objective lens, we can use the edge profile for a half-plane to approximate the edge profile of the curved fiber. In this approximation, the intensity as a function of the fiber radius is the same as the intensity along the $y$ axis for the half-plane calculation. We assumed a perfectly circular fiber. Figure 19 is the edge table of the simulated fiber image. 


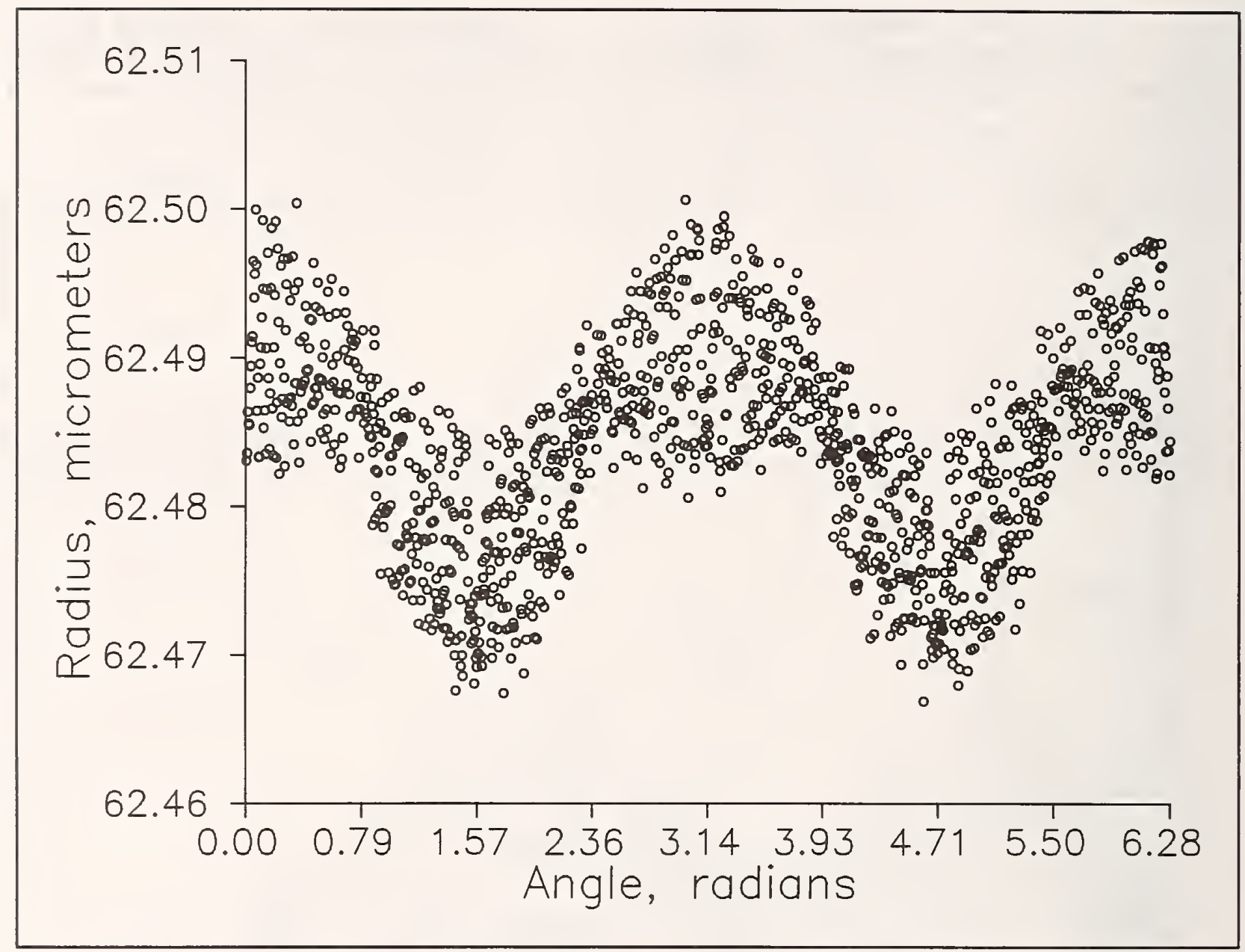

Figure 19. Simulated edge of a circular fiber.

Figure 19 shows that a circular fiber imaged with rectangular pixels theoretically appears noncircular with a noncircularity of $0.025 \%$; the major axis is horizontal. This apparent noncircularity is significant compared the $0.05 \%$ noncircularity of the fiber we have been using.

The radius we chose for the simulation was $62.5 \mu \mathrm{m}$. Figure 19 shows a $0.015 \mu \mathrm{m}$ offset between the apparent mean radius and the true radius. While this is significant, it is a part of the offset between the micrometer measurement and the average of the defocus runs and does not represent a source of error.

Figure 20 is the simulated edge table expanded about $1.57 \mathrm{rad}\left(90^{\circ}\right)$. Figure 20 has nonrandom oscillations at $1.57 \mathrm{rad}\left(90^{\circ}\right)$ and $4.71 \mathrm{rad}\left(270^{\circ}\right)$ similar to those in the real data (Figure 18). The magnitude of the nonrandom oscillations predicted by the simulation is smaller than what we actually observed in the real data. Further, the simulation predicts a similar result at $0 \mathrm{rad}$ and $3.14 \mathrm{rad}\left(180^{\circ}\right)$. We do not see this in the actual data at those angles. 


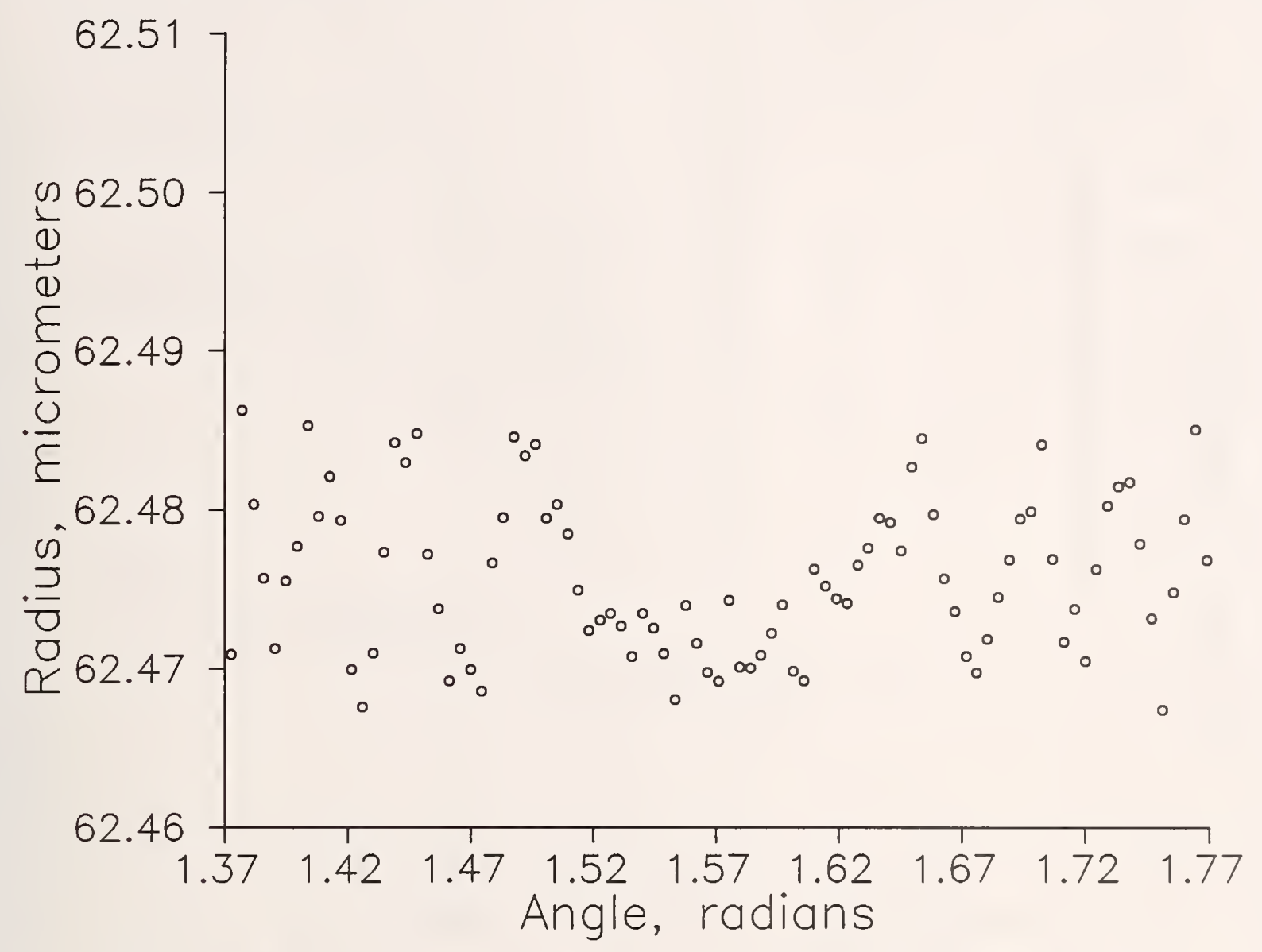

Figure 20. Oscillations of the image of a simulated edge.

The simulation also predicts an overall sine curve with maxima at $0^{\circ}$ and $180^{\circ}$ (Figure 19) and a noncircularity of $0.025 \%$. We do not see this in the real data either. Since yaw produces an effect just opposite to what is predicted by the simulation, a certain yaw angle could exactly cancel the overall sine due to pixelation. Since we adjusted the tilt to remove any sine curve which did not rotate with the fiber, we may have inadvertently corrected for the sine curve due to the pixelation.

The difference in magnitude between the simulated and real nonrandom oscillations indicates that we are not taking something into account. We looked at the data from the camera to check the shape of the intensity profile. Figure 21 compares the theoretical and real edge profiles (the position of the real profile on the horizontal scale is arbitrary). The edge profile of the real data is broader than that of the theoretical calculation. 


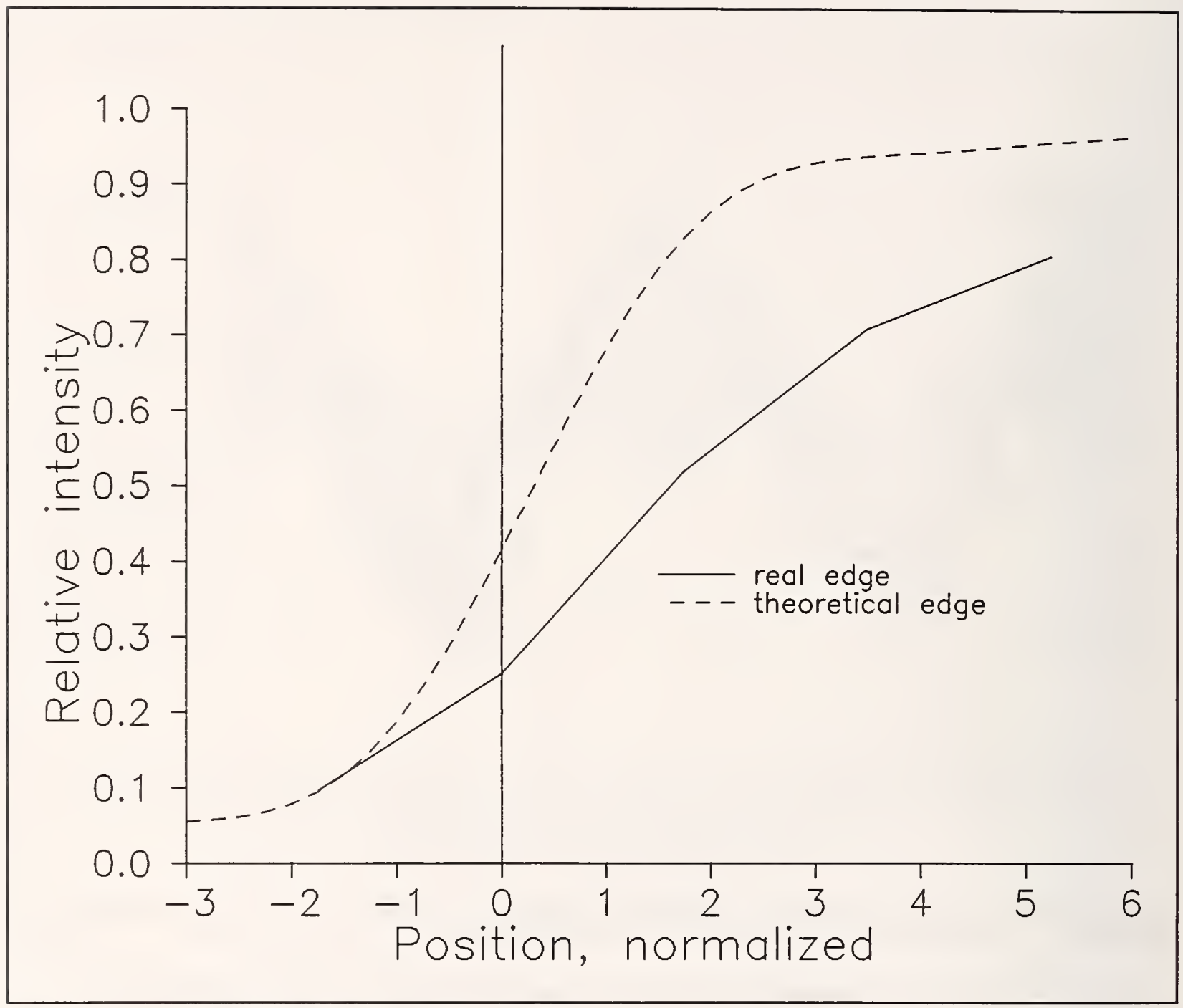

Figure 21. Theoretical and real edge profiles

1 resolution limit $=3.14$.

The difference between the shapes of the measured and theoretical edge profiles probably accounts for the difference in the magnitudes of the nonrandom oscillations. It probably also means that the intensity we have been using for edge detection is incorrect; this could cause all or part of the $0.19 \mu \mathrm{m}$ error in the mean diameter.

The measured edge profile is probably broader than the theoretical because of aberrations in the objective lens. Our calculation did not include lens aberrations, which increase the radius of the Green's function of the system and therefore change the mutual coherence function. In addition, we assumed a mutually incoherent source, even though the white plate was illuminated by a source that was itself not wholly incoherent. These factors make our calculation quantitatively incorrect and defeated our attempt to measure the diameter of the fiber without recourse to the calibration artifact.

One of the final stages in the camera is a low-pass filter. This low-pass filter operates on the electronic signal before it gets to the frame digitizer. This filter sees the outgoing data as a long string of voltage peaks. The net effect is a low-pass filtering of each horizontal line of pixels but not of the vertical columns of pixels. We assumed that the low-pass filter is a simple resistor-capacitor filter whose step response is an exponential decay with time. The cutoff frequency of the filter corresponds 


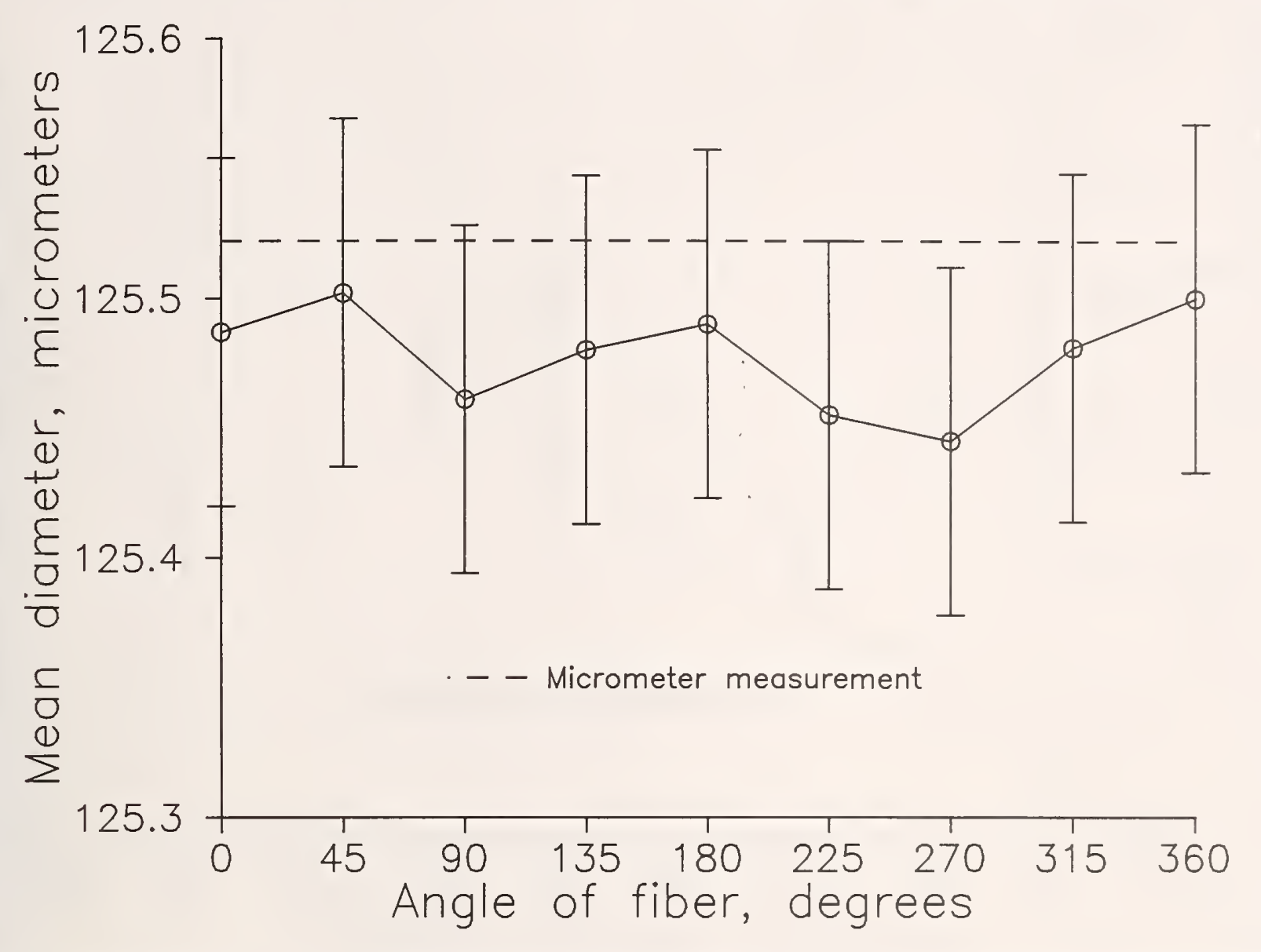

Figure 30. Mean diameter as a function of fiber rotation angle.

Figure 31 shows noncircularity as a function of fiber rotation angle. The noncircularity oscillates about the micrometer value as the fiber rotation angle changes. This could indicate that the beam splitter mount has moved and created a tilt angle. 


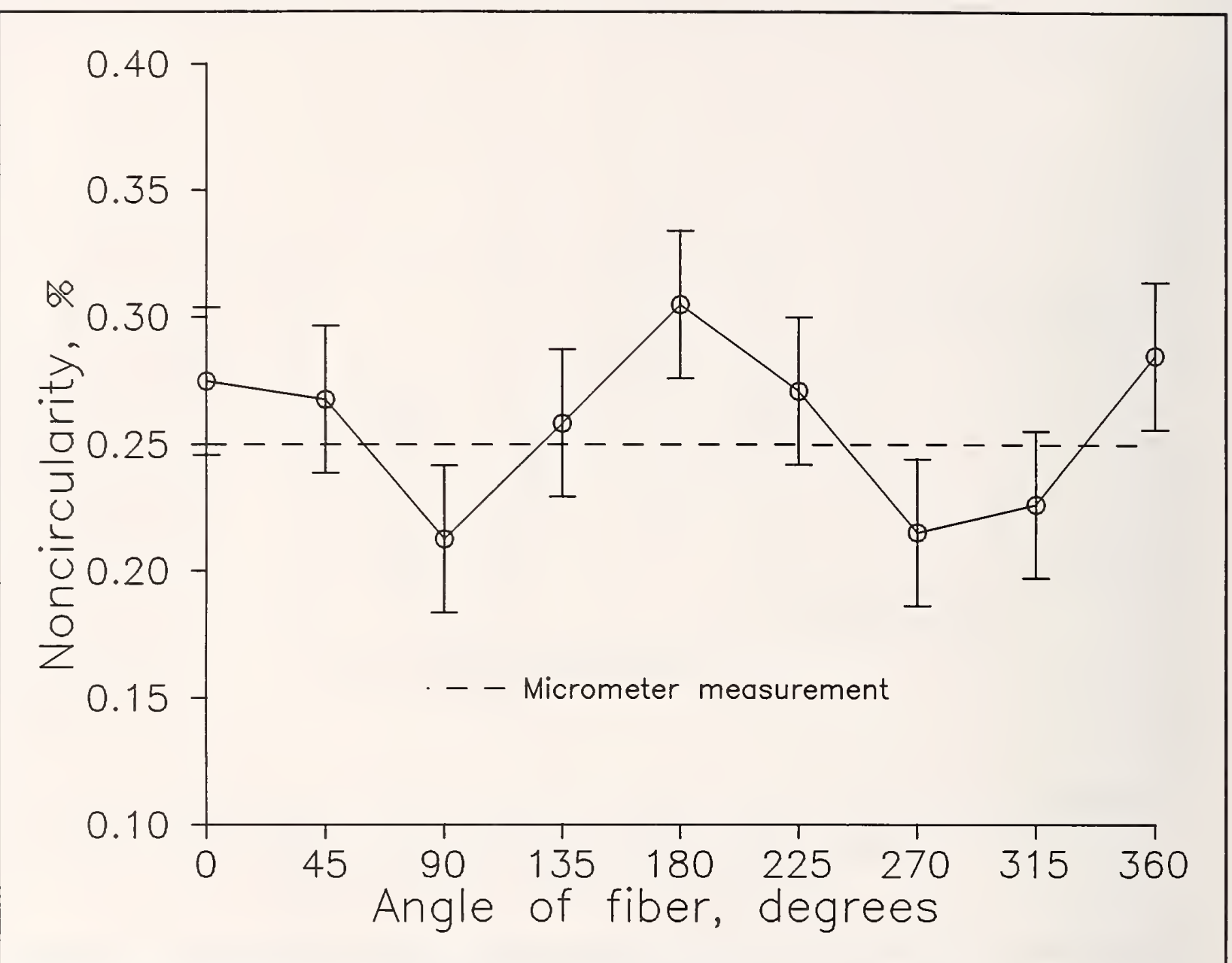

Figure 31. Noncircularity as a function of fiber rotation angle.

Figure 32 shows the angle of the major axis as a function of fiber rotation angle. The agreement of these data is very good. 


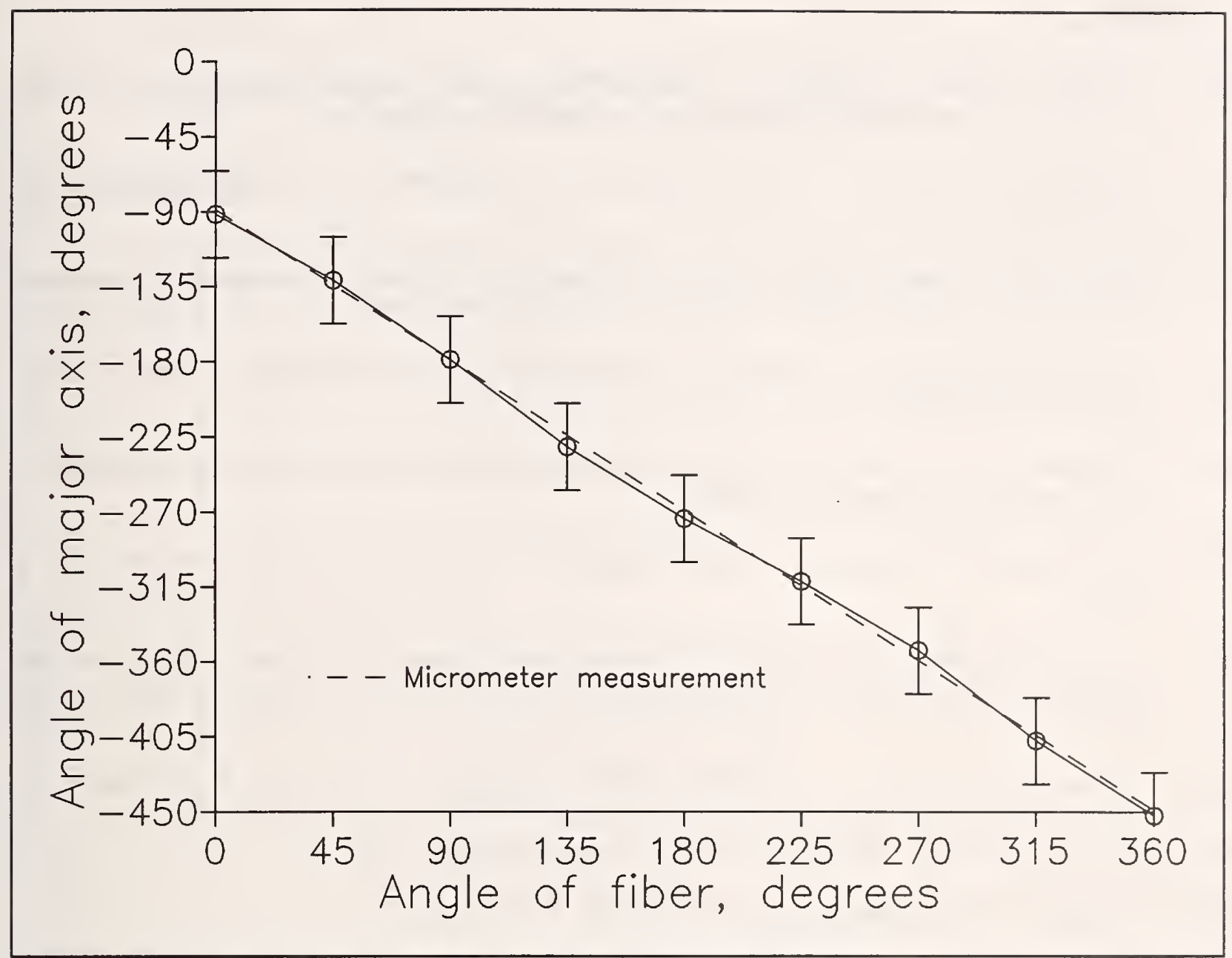

Figure 32. Angle of major axis as a function of fiber rotation angle.

\section{Conclusions}

With an SRM fiber as a calibration artifact, we succeeded in making mean diameter measurements with an uncertainty less than $0.1 \mu \mathrm{m}$. However, we did not succeed in making the measurements absolutely, that is, without using the calibration artifact.

The difference between a real edge profile and the theoretical edge profile in Figure 22 shows that we have not calculated the edge-finding intensity in a realistic manner. This is probably the result of aberrations in the lens, which was an inexpensive microscope objective. A better lens might reduce the error to less than the uncertainty of the measurements. Alternatively, the calculation of the edge profile could be altered to take aberrations into account.

The $3 \sigma$ uncertainty of $0.07 \mu \mathrm{m}$ in our measurements of mean diameter could be reduced by a better focusing method. Ideally, we would like computer-controlled motorized micrometers to move the fiber. The computer could continuously adjust the micrometers to produce the sharpest edge possible. Motorized micrometers alone might improve focusing simply because placing our hands on the instrument moves the fiber noticeably. Likewise, the $3 \sigma$ uncertainty of $0.03 \%$ in the noncircularity could be improved by motor-driven tilt adjustments with computer feedback.

Finally, details of the software used to create the edge tables, fit the ellipses, calculate the centers, and so on are provided in [13]. 


\section{References}

[1] Young, M.; Hale, P.D.; Mechels, S. "Optical fiber geometry: accurate measurement of cladding diameter," J. Res. Natl. Inst. Stand. Technol. 98(2): 203; 1993.

[2] Mechels, S.; Young, M. "Video microscope with submicrometer resolution," Appl. Opt. 30: 2202-2211; 1991.

[3] Mamileti, L.; Wang, C.J.; Young, M.; Vecchia, D.F. "Optical fiber geometry by gray-scale analysis with robust regression," Appl. Opt. 31: 4182-4185; 1992.

[4] Young, M.; Oates, C.W. "Microscope objectives, cover slips, and spherical aberration," Appl. Opt. 26: 2043; 1987.

[5] Gilblom, D.L. "Cameras for industrial sensing: this generation and the next," Proc. Soc. PhotoOpt. Instrum. Eng. 959: 66-86; 1988.

[6] Rousseeuw, P.J.; Leroy, A.M. Robust Regression \& Outlier Detection (John Wiley \& Sons); 1987.

[7] Vecchia, D., Wang, C.J.; Young, M. Outlier-resistant fitting of gray-scale images illustrated by optical fiber geometry, 1993 Measurement Science Conference, Los Angeles; January 21-22, 1993.

[8] Beran, M.J.; Parrent, G.B., Jr. Theory of Partial Coherence (Prentice-Hall, Inc.); 1964.

[9] Born, M.; Wolf, E. Principles of Optics (Pergamon Press); 1975.

[10] Goodman, J.W. Statistical Optics (John Wiley \& Sons); 1985.

[11] Young, M.; Hale, P.D. "Off-axis illumination and its relation to partial coherence," submitted to Amer. J. Phys.; 1993.

[12] Gaskill, J.D. Linear Systems, Fourier Transforms, and Optics (John Wiley \& Sons); 1978.

[13] Wang, C.M.; Vecchia, D.F.; Young, M.; Brilliant, N.A. Software for Performing Gray-Scale Measurements of Optical Fiber End Faces, Natl. Inst. Stand. Technol. Tech. Note 1370: in press.

[14] Dahlquist, G.; Björck, A. Numerical Methods (Prentice Hall, Inc.); 1974.

[15] Stoer, J.; Bulirsch, R. Introduction to Numerical Analysis (Springer Verlag); 1980.

[16] Golub, G.H.; Welsch, J.H. "Calculation of Gaussian quadrature rules," Math. Comp. 23: 221-230; 1969.

[17] Abramowitz, M.; Stegun, I.A. Handbook of Mathematical Functions, Nat. Bur. Stand. (U.S.) AMS 55; 1964.

[18] Wittmann, R.C.; Alpert, B.K. "An integral occuring in coherence theory," SIAM Rev. 36: December, 1994. 


\section{Appendix. \\ Evaluation of the Integrals of Partial Coherence}

\section{Problem Statement}

A uniform disk source of incoherent, quasimonochromatic light obstructed by a half-plane and focused by a lens system with circular stop produces image plane intensity at a point $(a, b)$ modeled using the van Cittert-Zernike approximation [9], [10] as

$$
I(a, b)=\int_{-\infty}^{\infty} d x \int_{0}^{\infty} d y \int_{-\infty}^{\infty} d w \int_{0}^{\infty} d z s\left(\alpha r_{1}\right) s\left(r_{2}\right) s\left(r_{3}\right) e^{i \beta\left(r_{4}{ }^{2}-r_{5}{ }^{2}\right)}
$$

where the sombrero function $s$ is defined by the formula

$$
s(r)=\frac{J_{1}(r)}{r},
$$

$J_{1}$ is the Bessel function of order 1 , and

$$
\begin{aligned}
& r_{1}=\sqrt{(x-w)^{2}+(y-z)^{2}}, \\
& r_{2}=\sqrt{(x-a)^{2}+(y-b)^{2}}, \\
& r_{3}=\sqrt{(w-a)^{2}+(z-b)^{2}}, \\
& r_{4}=\sqrt{x^{2}+y^{2}} \\
& r_{5}=\sqrt{w^{2}+z^{2}} .
\end{aligned}
$$

The intensity at $(a, b)$ without the half-plane obstruction is similarly modeled as

$$
N(a, b)=\int_{-\infty}^{\infty} d x \int_{-\infty}^{\infty} d y \int_{-\infty}^{\infty} d w \int_{-\infty}^{\infty} d z s\left(\alpha r_{1}\right) s\left(r_{2}\right) s\left(r_{3}\right) e^{i \beta\left(r_{4}{ }^{2}-r_{5}{ }^{2}\right)} .
$$

and the relative intensity is $I(a, b) / N(a, b)$.

The constants $\alpha$ and $\beta$ are determined by the geometry of the experimental apparatus. For a point source of (coherent) light, $\alpha=\beta=0$, and the integrals separate. In the experimental setup of interest, $\alpha$ had a value near 1 and $\beta$ was roughly $10^{-4}$. The relative intensity was desired for $a=0$ and a range of real values for $b$.

The computation of $I(a, b)$ using standard software would be prohibitively expensive. We describe several simplifications which reduce the number of operations enough to make the computation possible on a workstation.

\section{Numerical Method}

We observe that for small $\Delta b, I(a, b)$ and $I(a, b+\Delta b)$ differ by an integral that is easier to compute than either integral for $I$ alone. More precisely, $\partial I(a, b) / \partial b$ is an integral in three dimensions (rather than 
four):

$$
\begin{aligned}
\frac{\partial}{\partial b} I(a, b)= & \lim _{\delta \rightarrow 0} \delta^{-1}(I(a, b+\delta)-I(a, b)) \\
= & \lim _{\delta \rightarrow 0} \delta^{-1}\left\{\int_{-\infty}^{\infty} d x \int_{-\delta}^{\infty} d y \int_{-\infty}^{\infty} d w \int_{-\delta}^{\infty} d z s\left(\alpha r_{1}\right) s\left(r_{2}\right) s\left(r_{3}\right) \times\right. \\
& \left.e^{i \beta\left(x^{2}+(y+\delta)^{2}-w^{2}-(z+\delta)^{2}\right)}-I(a, b)\right\} \\
= & \lim _{\delta \rightarrow 0} \delta^{-1}\left\{2 \int_{-\infty}^{\infty} d x \int_{0}^{\infty} d y \int_{-\infty}^{\infty} d w \int_{-\delta}^{0} d z s\left(\alpha r_{1}\right) s\left(r_{2}\right) s\left(r_{3}\right) \times\right. \\
& +\int_{-\infty}^{i \beta\left(x^{2}+(y+\delta)^{2}-w^{2}-(z+\delta)^{2}\right)} d x \int_{0}^{\infty} d y \int_{-\infty}^{\infty} d w \int_{0}^{\infty} d z s\left(\alpha r_{1}\right) s\left(r_{2}\right) s\left(r_{3}\right) \times \\
= & \left.2 \int_{-\infty}^{\infty} d x \int_{0}^{\infty} d y \int_{-\infty}^{\infty} d w s\left(\alpha r_{1}\right) s\left(r_{2}\right) s\left(r_{3}\right) e^{i \beta\left(r_{4}{ }^{2}-r_{5}{ }^{2}\right)}\right|_{z=0} \\
& +\int_{-\infty}^{\infty} d x \int_{0}^{\infty} d y \int_{-\infty}^{\infty} d w \int_{0}^{\infty} d z s\left(\alpha r_{1}\right) s\left(r_{2}\right) s\left(r_{3}\right) e^{i \beta\left(r_{4}{ }^{2}-r_{5}{ }^{2}\right)} 2 i \beta(y-z) \\
= & \left.2 \int_{-\infty}^{\infty} d x \int_{0}^{\infty} d y \int_{-\infty}^{\infty} d w s\left(\alpha r_{1}\right) s\left(r_{2}\right) s\left(r_{3}\right) e^{i \beta\left(r_{4}{ }^{2}-r_{5}{ }^{2}\right)}\right|_{z=0} \cdot
\end{aligned}
$$

The same method can be used to show

$$
\frac{\partial}{\partial a} N(a, b)=\frac{\partial}{\partial b} N(a, b)=0
$$

so $N$ depends only on $\alpha$ and $\beta$.

Simpson's quadrature rule,

$$
\int_{b}^{b+h} f(x) d x \approx \frac{h}{6}(f(b)+4 f(b+h / 2)+f(b+h)),
$$

is employed to compute an approximation $\tilde{I}(a, b)$ to the intensity $I(a, b)$ :

$$
\tilde{I}(a, b+h)=\tilde{I}(a, b)+\frac{h}{6}\left(\frac{\partial}{\partial b} I(a, b)+4 \frac{\partial}{\partial b} I(a, b+h / 2)+\frac{\partial}{\partial b} I(a, b+h)\right),
$$

for a chosen step size $h$. This approximation's error is of order $O\left(h^{4}\right)$ (fourth order).

We approximate the integral (9) for $\partial I / \partial b$ by restricting the domain to be finite,

$$
I_{t}=\left.2 \int_{-t}^{t} d x \int_{0}^{t} d y \int_{-t}^{t} d w s\left(\alpha r_{1}\right) s\left(r_{2}\right) s\left(r_{3}\right) e^{i\left(\beta\left(r_{4}{ }^{2}-r_{5}{ }^{2}\right)\right.}\right|_{z=0},
$$


which allows the integration to be performed to high accuracy. To obtain an estimate of $I_{\infty}$, we make the extrapolation assumption that $I_{t}$ has the form

$$
I_{t}=I_{\infty}+\frac{\alpha_{1}}{t}+\frac{\alpha_{2}}{t^{2}}+\cdots
$$

We truncate the right side of (14) at three terms, obtain 3 linear equations in the 3 unknowns $I_{\infty}, \alpha_{1}$, and $\alpha_{2}$ by substituting computed values for $I_{t}, I_{2 t}$, and $I_{4 t}$, and then solve for the unknowns. This extrapolation procedure can be expected to be reliable if $t$ is chosen large enough that $I_{t}-I_{2 t}$ is small relative to $I_{t}$ and $I_{t}-I_{2 t} \approx 2\left(I_{2 t}-I_{4 t}\right)$. Our computations indicate that these conditions are met for the parameter values of interest, $\alpha \approx 1, \beta \approx 10^{-4}, a=0$, and $-6<b<6$, provided that $t \geq 20$. Based on the computations, we believe that this extrapolation decreases the error in approximating $I_{\infty}$ from $O\left(t^{-1}\right)$ to $O\left(t^{-3}\right)$; that is, it yields third-order convergence.

We compute $I_{t}$ with Gaussian quadrature [14], [15]. In particular, we use Gauss-Legendre quadrature with $n(t)$ nodes in $y$ and $2 n(t)$ nodes in $x$ and $w$, where

$$
n(t)= \begin{cases}t, & 0 \leq \alpha \leq 1 \\ \alpha t, & \alpha>1\end{cases}
$$

Gaussian-Legendre quadrature approximates an integral by an linear combination of values of the integrand,

$$
\int_{-1}^{1} f(x) d x \approx \sum_{i=1}^{n} w_{i} f\left(x_{i}\right),
$$

where the nodes $x_{1}, \ldots, x_{n}$ and weights $w_{1}, \ldots, w_{n}$ are determined so that (16) is exact if $f$ is a polynomial of degree up to $2 n-1$. (The nodes coincide with the roots of the Legendre polynomial of degree $n$.) We determine the nodes and weights using standard software [16]. These quadratures converge extremely rapidly for any infinitely continuously differentiable function $f$; in our case the quadratures are accurate to machine precision (15 digits).

The integrand of $I_{t}$ involves the Bessel function $J_{1}$, which we compute from [17]

$$
J_{n-1}(x)=\frac{2 n}{x} J_{n}(x)-J_{n+1}(x),
$$

applied in order of decreasing $n$, combined with the normalization equation

$$
1=J_{0}(x)+2 J_{2}(x)+2 J_{4}(x)+2 J_{6}(x)+\cdots .
$$

Since the values of $s(x)=J_{1}(x) / x$ are required repeatedly, we tabulate this function at equispaced values of $x$ from 0 to the maximum value required $(\sqrt{5} t \max \{\alpha, 1\})$ and obtain the function at required values of $x$ by fourth-order interpolation. The table is set up so that the interpolation operation requires only three multiplications and three additions. The tabular increment of $x$ of 0.01 is chosen to yield 10 digit accuracy.

A straightforward computation of $I(a, b)$ proceeding directly from its definition (1) would be beyond our current computational resources. The result of our optimizations is that the required integrals can be computed on a workstation. We use a high-performance workstation that has peak performance of over 50 million floating-point operations per second; for a given value of $\alpha, \beta$, and $a$, we obtain $I(a, b)$ for $-6<$ $b<6$ in under an hour of computation time. The resulting accuracy is limited by the accuracy of the extrapolation; for $t=20$ the relative error is less than $10^{-3}$. 


\section{Supplementary Analytical Result}

The computed results for $\beta \approx 10^{-4}$ nearly coincide with those for $\beta=0$; the fact that this holds for the integrals can be shown analytically. As asserted above, $N(a, b)$ is actually independent of $(a, b)$, and for $\beta=0, N(\alpha)=N(a, b) /\left(2 \pi^{2}\right)$ has the values

$$
N(\alpha)= \begin{cases}1, & 0<\alpha \leq 1 \\ \alpha^{-2}, & \alpha>1,\end{cases}
$$

which Ron Wittmann has demonstrated analytically [18]. 


\section{Periodical}

Journal of Research of the National Institute of Standards and Technology-Reports NIST research and development in those disciplines of the physical and engineering sciences in which the Institute is active. These include physics, chemistry, engineering, mathematics, and computer sciences. Papers cover a broad range of subjects, with major emphasis on measurement methodology and the basic technology underlying standardization. Also included from time to time are survey articles on topics closely related to the Institute's technical and scientific programs. Issued six times a year.

\section{Nonperiodicals}

Monographs-Major contributions to the technical literature on various subjects related to the Institute's scientific and technical activities.

Handbooks-Recommended codes of engineering and industrial practice (including safety codes) developed in cooperation with interested industries, professional organizations, and regulatory bodies.

Special Publications-Include proceedings of conferences sponsored by NIST, NIST annual reports, and other special publications appropriate to this grouping such as wall charts, pocket cards, and bibliographies.

Applied Mathematics Series-Mathematical tables, manuals, and studies of special interest to physicists, engineers, chemists, biologists, mathematicians, computer programmers, and others engaged in scientific and technical work.

National Standard Reference Data Series-Provides quantitative data on the physical and chemical properties of materials, compiled from the world's literature and critically evaluated. Developed under a worldwide program coordinated by NIST under the authority of the National Standard Data Act (Public Law 90-396). NOTE: The Journal of Physical and Chemical Reference Data (JPCRD) is published bimonthly for NIST by the American Chemical Society (ACS) and the American Institute of Physics (AIP). Subscriptions, reprints, and supplements are available from ACS, 1155 Sixteenth St., NW, Washington, DC 20056.

Building Science Series-Disseminates technical information developed at the Institute on building materials, components, systems, and whole structures. The series presents research results, test methods, and performance criteria related to the structural and environmental functions and the durability and safety characteristics of building elements and systems.

Technical Notes-Studies or reports which are complete in themselves but restrictive in their treatment of a subject. Analogous to monographs but not so comprehensive in scope or definitive in treatment of the subject area. Often serve as a vehicle for final reports of work performed at NIST under the sponsorship of other government agencies.

Voluntary Product Standards-Developed under procedures published by the Department of Commerce in Part 10, Title 15, of the Code of Federal Regulations. The standards establish nationally recognized requirements for products, and provide all concerned interests with a basis for common understanding of the characteristics of the products. NIST administers this program in support of the efforts of privatesector standardizing organizations.

Consumer Information Series-Practical information, based on NIST research and experience, covering areas of interest to the consumer. Easily understandable language and illustrations provide useful background knowledge for shopping in today's technological marketplace.

Order the above NIST publications from: Superintendent of Documents, Government Printing Office, Washington, DC 20402.

Order the following NIST publications-FIPS and NISTIRs-from the National Technical Information Service, Springfield, VA 22161.

Federal Information Processing Standards Publications (FIPS PUB)-Publications in this series collectively constitute the Federal Information Processing Standards Register. The Register serves as the official source of information in the Federal Government regarding standards issued by NIST pursuant to the Federal Property and Administrative Services Act of 1949 as amended, Public Law 89-306 (79 Stat. 1127), and as implemented by Executive Order 11717 (38 FR 12315, dated May 11, 1973) and Part 6 of Title 15 CFR (Code of Federal Regulations).

NIST Interagency Reports (NISTIR)-A special series of interim or final reports on work performed by NIST for outside sponsors (both government and non-government). In general, initial distribution is handled by the sponsor; public distribution is by the National Technical Information Service. Springfield, VA 22161, in paper copy or microfiche form. 
U.S. Department of Commerce

National Institute of Standards and Technology

325 Broadway

Boulder, Colorado 80303-3328

Official Business

Penalty for Private Use, $\$ \mathbf{3 0 0}$ 